\title{
LECTURES ON INDICES AND RELATIVE INDICES ON CONTACT AND CR-MANIFOLDS
}

\author{
BY CHARLES L. EPSTEIN \\ PHILADELPHIA, PA
}

\section{CONTENTS}

1. Introduction 1

2. Fredholm operators and Toeplitz operators on the circle 2

3. Contact and CR-manifolds 5

4. CR-functions and a generalization of Toeplitz operators 10

5. Pseudodifferential operators, symbols and the radial compactification of the $\begin{array}{ll}\text { cotangent bundle } & 11\end{array}$

6. Parabolic compactifications for contact manifolds $\quad 16$

7. The Heisenberg calculus 18

8. Application of the Heisenberg calculus to several complex variables 22

9. The quantum harmonic oscillator and $\square_{b} \quad 25$

10. Fields of harmonic oscillators $\quad 27$

11. Vector bundle coefficients and the Atiyah-Singer index theorem 31

12. The Boutet de Monvel index formula 34

13. An index theorem for the Heisenberg calculus 36

14. The structure of the higher eigen-projections 37

15. Grauert tubes and the Atiyah-Singer index theorem 42

16. The contact degree and index of FIOs 44

$\begin{array}{ll}\text { References } & 46\end{array}$

\section{INTRODUCTION}

These are notes for lectures that I presented at the summer school held at CIRM in Luminy, June 29-July 9, 1999 and Woods Hole, MA October 1999. I have added additional material to make the notes more comprehensible and self contained as well as some additional results in the last two sections. The aim of the lectures was to provide sufficient background to discuss recent work, done with Richard Melrose and Gerardo Mendoza on index formulæ for Fredholm operators in the Heisenberg calculus. Complete proofs of the results described in these lectures and considerable extensions of these results are found in the monograph by Melrose and myself, [14].

Acknowledgments

I would like to thank the organizers of the summer school in Marseilles, B. Coupet, J. Merker and A. Shukov and the meeting in Woods Hole, Nils Tongring and Dennis Sullivan for giving me the opportunity to present these lectures. I would also like to thank Hyunsuk Kang for very kindly providing a careful transcript of the original lectures. Finally I would like to thank my collaborators,

Date: May 13, 2002.

Research supported in part by the NSF. 
Richard Melrose and Gerardo Mendoza for allowing me to write this expository account of our joint work.

\section{FredHOLM OPERATORS AND TOEPLITZ OPERATORS ON THE CIRCLE}

Our story begins with a classical theorem which I, somewhat incorrectly, call "The Szegó index theorem." The history of this result is unclear, it appears that the first such result was by proved in 1921 by Fritz Noether in [30]. Let $S^{1}$ be the unit circle in the complex plane. A function or distribution on the unit circle can be expanded in a Fourier series. A distribution is the boundary value of a holomorphic function on the unit disk if and only if its negative Fourier coefficients all vanish. Define the sub-space $H^{2}\left(S^{1}\right) \subset$ $L^{2}\left(S^{1}\right)$ by

$$
H^{2}\left(S^{1}\right)=\left\{f \in L^{2}\left(S^{1}\right) \mid f=\sum_{n=0}^{\infty} a_{n} e^{i n \theta}\right\} .
$$

Let $\pi: L^{2}\left(S^{1}\right) \rightarrow H^{2}\left(S^{1}\right)$ be the orthogonal projection operator. If $f$ is a bounded measurable function then we define a Toeplitz operator $T_{f}: H^{2}\left(S^{1}\right) \rightarrow H^{2}\left(S^{1}\right)$ by

$$
T_{f} u=\pi f \pi(u) .
$$

The first $\pi$ is included as it is sometimes convenient to think of $T_{f}: L^{2}\left(S^{1}\right) \rightarrow H^{2}\left(S^{1}\right)$.

One can easily prove the estimate

$$
\left\|T_{f} u\right\|_{L^{2}} \leq\|f\|_{L^{\infty}}\|u\|_{L^{2}} .
$$

This estimate can be read in two different ways. As an operator acting on $u, T_{f}$ is a bounded operator with $\left\|T_{f}\right\|_{\text {op }} \leq\|f\|_{L^{\infty}}$. Since $T_{f}-T_{g}=T_{f-g}$ this implies that

$$
\left\|T_{f}-T_{g}\right\|_{\text {op }} \leq\|f-g\|_{L^{\infty}}
$$

provided $f, g \in L^{\infty}\left(S^{1}\right)$. Thus $f \mapsto T_{f}$ is a continuous map from $L^{\infty}\left(S^{1}\right)$ to bounded operators on $L^{2}\left(S^{1}\right)$ in the operator norm topology.

We are interested in a general class of operators called Fredholm operators.

Definition 1. Given Banach spaces $X$ and $Y$, a bounded linear operator $A: X \rightarrow Y$ is called Fredholm if it satisfies the following three conditions:

(a) The range of $A$ is closed as a subspace of $Y$,

(b) The dimension of the null space of $A$ is finite.

(c) The dimension of the co-kernel, $Y / A X$ is finite.

The Fredholm index is defined by the formula

$$
\text { Ind } A=\operatorname{dim}(\operatorname{ker} A)-\operatorname{dim}(\operatorname{coker} A) \text {. }
$$

If the index is non-zero then the operator $A$ is not invertible. On the other hand if Ind $A$ vanishes then there is a compact operator $K$ such that $A+K$ is invertible. For most linear operators it is very difficult to compute the dimension of either the kernel or the co-kernel but their difference, the Fredholm index is often computable. This is a reason that there is so much interest in Fredholm indices.

An operator $A$ is Fredholm if and only if there exists a bounded operator $B: Y \rightarrow X$ such that

$$
B A-I_{X}=K_{1}, A B-I_{Y}=K_{2}
$$

where $K_{1}, K_{2}$ are compact operators. In other words, Fredholm operators are operators which are invertible up to a compact error. In fact, $K_{1}$ and $K_{2}$ can be always taken to be trace class operators. In that case the index is given by

$$
\text { Ind } A=\operatorname{Tr}[B, A]=\operatorname{Tr}\left(K_{1}-K_{2}\right) \text {. }
$$


The trace of a commutator of finite rank, or more generally trace class operators is zero; the Fredholm index is a measure of the failure of this result for "almost invertible" operators acting on infinite dimensional spaces.

If $X$ and $Y$ are finite dimensional then

$$
\text { Ind } A=\operatorname{dim} X-\operatorname{dim} Y \text {. }
$$

In certain instances this formula is used metaphorically, in case $X$ and $Y$ are infinite dimensional, to define a renormalized "difference of the dimensions" of $X$ and $Y$. For example if $X$ and $Y$ are subspaces of a larger space and $A$ is an orthogonal projection then Ind $A$ often gives a reasonable definition for " $\operatorname{dim} X-\operatorname{dim} Y$." Care must be exercised as there are Fredholm operators from a space to itself with non-zero index.

We now recall the basic properties of Fredholm operators.

Lemma 1. For parts (a) and (b) $A$ and $B$ are Fredholm operators from $X$ to $Y$.

(a) If $A$ is Fredholm operator, then there exists $\epsilon>0$ such that if $\|B\|_{\mathrm{op}}<\epsilon$, then $A+B$ is also Fredholm operator and

$$
\text { Ind } A=\operatorname{Ind}(A+B) \text {. }
$$

The index is stable, so we do not need to have perfect knowledge of an operator to compute its index.

(b) If $A$ is Fredholm and $K: X \rightarrow Y$ is compact, then $A+K$ is Fredholm and

$$
\text { Ind } A=\operatorname{Ind}(A+K) \text {. }
$$

Changing a Fredholm operator by a compact operator does not change its index. This means that we are allowed perturbations of arbitrary norm so long as they are compact.

(c) If $A: X \rightarrow Y$ and $B: Y \rightarrow Z$ are Fredholm operators then so is $B \circ A$ and we have

$$
\text { Ind } B \circ A=\operatorname{Ind} B+\operatorname{Ind} A \text {. }
$$

(d) If $A: X \rightarrow Y$ is a Fredholm operator then $A^{*}: Y^{\prime} \rightarrow X^{\prime}$ is as well and

$$
\operatorname{Ind}\left(A^{*}\right)=-\operatorname{Ind}(A) \text {. }
$$

(e) If $t \rightarrow A_{t}$ is a 1-parameter family of operators for $t \in[0,1]$, continuous, with respect to the operator norm and $A_{t}$ is a Fredholm operator for each $t$, then Ind $A_{t}$ is constant. This is an immediate consequence of the fact that the index is a continuous, integer valued function.

The proofs of these statements and a good general discussion of Fredholm operators can be found in [21].

We now analyze Toeplitz operators acting on $L^{2}\left(S^{1}\right)$.

Proposition 1. If $f \in \mathcal{C}^{0}\left(S^{1}\right)$ and $f$ does not vanish, then $T_{f}$ is a Fredholm operator.

To prove this proposition we use the following lemma.

Lemma 2. If $f \in \mathcal{C}^{0}\left(S^{1}\right)$, then $\pi[\pi, f]$ is a compact operator.

Proof. If $f$ is an exponential polynomial, say, $f=\sum_{j=-N}^{N} a_{j} e^{i j t}$, then

$$
\pi[\pi, f]: L^{2}\left(S^{1}\right) \rightarrow H^{2}\left(S^{1}\right)
$$


is a finite rank operator. For $f \in \mathcal{C}^{0}\left(S^{1}\right)$ let $\sigma_{N} f$ be the $N^{\text {th }}$ Cesaro mean of the Fourier series of $f$. Recall that for a continuous function

$$
\lim _{N \rightarrow \infty}\left\|f-\sigma_{N} f\right\|_{L^{\infty}}=0 .
$$

Since $\pi$ is an orthogonal projection it follows from (3) that

$$
\left\|\pi\left[\pi, \sigma_{N} f\right]-\pi[\pi, f]\right\| \leq\left\|\sigma_{N} f-f\right\|_{L^{\infty}} \rightarrow 0 \text { as } N \rightarrow \infty .
$$

Hence $\pi[\pi, f]$ is the norm limit of finite rank operators and therefore compact.

Proof of Proposition. Since $f$ is non-vanishing the function $f^{-1} \in \mathcal{C}^{0}\left(S^{1}\right)$. As $\pi^{2}=\pi$ we have the identity:

$$
T_{f^{-1}} T_{f}=\pi f^{-1} \pi f \pi=\pi f^{-1} f \pi+\pi\left[f^{-1}, \pi\right] f \pi=\pi+K_{1},
$$

for $K_{1}$ a compact operator. Hence, $T_{f^{-1}}$ is the inverse of $T_{f}$ up to a compact error. Similarly we have $T_{f} T_{f^{-1}}=\pi+K_{2}$ for another compact operator $K_{2}$.

The next task is to compute the Ind $T_{f}$. Associated to a non-vanishing complex valued function is a topological invariant called the winding number. Orient $S^{1} \subset \mathbb{C}$ so that counterclockwise is the positive direction and let $\left[S^{1}\right]$ denote a positive generator for $H_{1}\left(S^{1} ; \mathbb{Z}\right)$ and $\left[\mathbb{C}^{*}\right]$ the counterclockwise unit circle thought of as a generator for $H_{1}\left(\mathbb{C}^{*} ; \mathbb{Z}\right)$.

Definition 2. Let $f: S^{1} \rightarrow \mathbb{C} \backslash\{0\}$ be a continuous map then $f_{*}\left[S^{1}\right]=k\left[\mathbb{C}^{*}\right]$ for an integer $k$. The number $k$ is the winding number of $f$, which we denote $\mathrm{w}_{\#}(f)$.

An example of a function with winding number $k$ is $f_{k}\left(e^{i \theta}\right)=e^{i k \theta}$.

Exercise 1. Show that Ind $T_{f_{k}}=-k$.

From the definition it is clear that the winding number of $f$ only depends on the homotopy class of the map $f: S^{1} \rightarrow \mathbb{C}^{*}$. In fact the winding number labels the connected components of the homotopy classes of these maps: $\mathrm{w}_{\#}(f)=\mathrm{w}_{\#}(g)$ if and only if $f$ can be homotoped to $g$. Note that any map $f$ into $\mathbb{C}^{*}$ is homotopic to the map $f|f|^{-1}$.

Exercise 2. Using the fact that $\mathbb{R}$ is the universal cover of $S^{1}$, prove that $\mathrm{w}_{\#}(f)=k$ if and only if $f$ is homotopic to $f_{k}$.

Suppose that $f, g: S^{1} \rightarrow \mathbb{C}^{*}$ then we have a formula for the winding of the product

$$
\mathrm{w}_{\#}(f \cdot g)=\mathrm{w}_{\#}(f)+\mathrm{w}_{\#}(g) .
$$

It is a simple consequence of Lemma 2 that $T_{f} \circ T_{g}=T_{f g}+K$ where $K$ is a compact operator. Note the similarity between this formula and (6). The index theorem for Fredholm Toeplitz operators is:

Theorem 1 (Szegó index theorem). If $f \in \mathcal{C}^{0}\left(S^{1}\right)$ and $f\left(e^{i \theta}\right) \neq 0$ for all $\theta$, then $T_{f}$ is a Fredholm operator, and

$$
\text { Ind } T_{f}=-\mathrm{w}_{\#}(f) \text {. }
$$

Note that the left hand side is an analytic quantity, whereas the right is purely topological. This is the pattern for all index theorems: an analytically defined invariant is shown to equal a topological invariant. One then tries to find a usable formula for the topological quantity. In the case at hand we have

Proposition 2. If $f: S^{1} \rightarrow \mathbb{C}^{*}$ is once differentiable then

$$
\mathrm{w}_{\#}(f)=\frac{i}{2 \pi} \int_{0}^{2 \pi} \frac{f^{\prime}\left(e^{i \theta}\right)}{f\left(e^{i \theta}\right)} d \theta .
$$


Exercise 3. Prove this formula

Proof of the theorem. Let $k=\mathrm{w}_{\#}(f)$, then $e^{-i k \theta} f\left(e^{i \theta}\right)$ has winding number zero. As stated above, a map with winding number zero is homotopic to a constant map. Hence there exists a continuous map $F_{t}\left(e^{i \theta}\right),\left(t, e^{i \theta}\right) \in[0,1] \times S^{1}$, such that

$$
F_{t}: S^{1} \rightarrow \mathbb{C} \backslash\{0\}, \text { with } F_{0}=e^{-i k \theta} f, F_{1}=1 .
$$

The 1-parameter family of maps $G_{t}=e^{i k \theta} F_{t}\left(e^{i \theta}\right)$ is then a homotopy between $f$ and $f_{k}$. Since $G_{t}$ is non-vanishing for each $t$ it follows that $T_{G_{t}}$ is a continuous family of Fredholm operators. From the homotopy invariance of the index we conclude that

$$
\text { Ind } T_{f_{k}}=\operatorname{Ind} T_{f} \text {. }
$$

From the exercise above we know that $\operatorname{Ind} T_{f_{k}}=-k$, which completes the proof of the theorem.

Let us summarize the elements of the above argument.

(a) We have a family of operators $\left\{T_{f}: f \in \mathcal{C}^{0}\left(S^{1}\right)\right\}$ parameterized by a "symbol" $f$.

(b) We have an algebraic condition on $f$ which implies $T_{f}$ is Fredholm; in this case: $f \neq 0$.

(c) Thinking of $f$, a non-vanishing symbol, as a map we define a topological invariant; in this case minus the winding number.

(d) Using the invariance properties of Fredholm index and the topological invariant, we show they are equal.

An important but, simple generalization of Toeplitz operators is to use "symbols," $f$ which take values in $n \times n$ complex matrices. Let $\pi_{n}$ denote the operator $\pi \otimes \operatorname{Id}_{n}$ acting on $n$-vector valued functions on $S^{1}, L^{2}\left(S^{1} ; \mathbb{C}^{n}\right)$. We define a matrix-Toeplitz operator by setting

$$
T_{f}=\pi_{n} f \pi_{n}
$$

Using Lemma 2 one easily establishes that if $f$ takes values in $\mathrm{Gl}_{n}(\mathbb{C})$ then $T_{f^{-1}}$ is an inverse for $T_{f} \uparrow_{\text {range } \pi_{n}}$, up to a compact error and therefore in this case $T_{f}: \operatorname{range} \pi_{n} \rightarrow$ range $\pi_{n}$ is Fredholm. The cohomology group $H_{1}\left(\mathrm{Gl}_{n} ; \mathbb{Z}\right)$ is again one dimensional and so we have an analogous definition for the winding number of $f: S^{1} \rightarrow \mathrm{Gl}_{n}(\mathbb{C})$. With this understood, the index theorem for a matrix Toeplitz operator is exactly the same as for a scalar Toeplitz operator. If the symbol is differentiable then the index formula reads

$$
\operatorname{Ind}\left(T_{f}\right)=\frac{i}{2 \pi} \int_{0}^{2 \pi} \operatorname{Tr}\left[f^{-1} f^{\prime}\right] d \theta .
$$

To get a scalar quantity we need to take a matrix trace. A bit more invariantly, matrix Toeplitz operators act on the sections of oriented vector bundles over the circle. For further discussion of these results see $[15,26]$.

Exercise 4 (More challenging exercise). Prove the matrix-Toeplitz index theorem.

\section{CONTACT AND CR-MANIFOLDS}

The archetypal index theorem that most people are familiar with is the Riemann-Roch theorem. One can actually do large parts of the subject of index theory from a slightly different point of view using the Szegő index theorem as the starting point. The RiemannRoch theorem is a theorem about complex curves. In particular it pertains to manifolds with even dimension. The Szegó index theorem is for a 1-dimensional manifold. We now consider how to generalize the definition of a Toeplitz operator. The correct context for 
this analysis turns out to be a contact manifold. Contact manifolds are odd-dimensional, and like the circle, often arise as the boundaries of complex manifolds.

Let $M$ be a $(2 n+1)$-dimensional manifold. Let $\mathcal{H} \subset T M$ denote a co-dimension one sub-bundle, briefly a hyperplane field. Locally we can choose a one form $\theta$ such that $H=\operatorname{ker} \theta$.

Definition 3. A hyperplane field $\mathcal{H} \subset T M$ defines a contact structure if and only if $\theta \wedge[d \theta]^{n}$ is nowhere vanishing.

A contact structure is the opposite of a foliation. It is a nowhere integrable hyperplane field. The contact field is co-orientable if the line bundle $T M / \mathcal{H}$ is orientable. A coorientation for $\mathcal{H}$ is a therefore global, smooth choice of positive half spaces in $T M \backslash \mathcal{H}$. A 1-form annihilating $\mathcal{H}$ is positive if it positive on the positive half spaces. If $\mathcal{H}$ is coorientable then it is the kernel of a globally defined 1-form, called a contact form. If $\theta$ is a positive contact form then, for any smooth function $\rho, e^{\rho} \theta$ is as well. The standard model for a contact manifold is $\mathbb{R}^{2 n+1}$ with the contact structure defined by the 1 -form:

$$
\theta_{0}=d t+\frac{1}{2} \sum_{j=1}^{n}\left[x_{j} d y_{j}-y_{j} d x_{j}\right] \text {. }
$$

There are two basic classes of examples of contact manifolds. One comes from classical mechanics and the other from complex analysis. We begin with the first class. Let $X$ be an $(n+1)$-dimensional, real manifold. Its cotangent bundle $T^{*} X$ is a symplectic manifold with symplectic form given by

$$
\omega=\sum_{i=1}^{n+1} d x_{i} \wedge d \xi_{i}
$$

where $\left(x_{1}, \ldots, x_{n+1}\right)$ are local coordinates and $\left(\xi_{1}, \ldots, \xi_{n+1}\right)$ are fiber coordinates defined by the local trivialization, $\left\{d x_{1}, \ldots, d x_{n+1}\right\}$, of $T^{*} X$. It is obvious that the 2 -form $\omega$ is closed. It is a little less apparent, but nonetheless true that $\omega$ is globally defined and does not depend on the choice of coordinates. This makes the cotangent bundle into a symplectic manifold. There is an action of $\mathbb{R}^{+}=(0, \infty)$ on $T^{*} X$ by

$$
M_{\lambda}(x, \xi):=(x, \lambda \xi) .
$$

We see that $M_{\lambda}^{*}(\omega)=\lambda \omega$. Hence $T^{*} X$ is called a conic, symplectic manifold because the symplectic form is homogeneous of degree one. Let

$$
S^{*} X=T^{*} X \backslash\{0\} / \mathbb{R}_{+}
$$

be the co-sphere bundle. If $g$ denotes a Riemannian metric on $T^{*} X$ then we define $S_{g}^{*} X$ to be the set of vectors of unit length with respect to this metric. This is called a unit cosphere bundle. For any choice of $g$ the restriction of the quotient map $q_{g}: S_{g}^{*} X \rightarrow S^{*} X$ is a diffeomorphism. Note that the quotient by the $\mathbb{R}^{+}$-action is canonical, while the choice of metric is not. Let $V=\sum_{i=1}^{n+1} \xi_{i} \partial_{\xi_{i}}$ be the generator of the $\mathbb{R}^{+}$-action. If we take the interior product of $V$ with $\omega$, we get the 1-form:

$$
\alpha=i_{V} \omega=-\sum_{i=1}^{n+1} \xi_{i} d x_{i} .
$$

A simple calculation shows that $d \alpha=\omega$.

Restricting $\alpha$ to $S_{g}^{*} X$ defines a nowhere vanishing form, let $\mathcal{H}_{g} \subset T S_{g}^{*} X$ denote its null-space. Using the fact that $V$ is transverse to $S_{g}^{*} X$ one easily shows that $\alpha \wedge[d \alpha]^{n}$ is nowhere vanishing and hence $\mathcal{H}_{g}$ is a contact structure. Since $\alpha$ is homogeneous of degree 
1 under the $\mathbb{R}^{+}$-action and annihilates the infinitesimal generator it defines a conformal class of one forms on the quotient $S^{*} X$. It is again a simple computation to show that the null-space of this conformal class of one forms, $\mathcal{H}$ defines a contact structure on the cosphere bundle. Indeed the quotient map $q_{g}: S_{g}^{*} X \rightarrow S^{*} X$ is a contact diffeomorphism: $q_{g *}: \mathcal{H}_{g} \rightarrow \mathcal{H}$.

From a conic, symplectic manifold we have obtained a contact manifold in a canonical way. The process can be reversed. Suppose that $(M, \mathcal{H})$ is a co-oriented contact manifold. Let $\Lambda_{\mathcal{H}}$ be the ray sub-bundle of $T^{*} M$ consisting of positive contact 1 -forms. If $\theta$ is a choice of contact form then $(p, \lambda) \mapsto \lambda \theta_{p}$ defines a diffeomorphism between $M \times \mathbb{R}^{+}$ and $\Lambda_{\mathcal{H}}$. The symplectic structure on $T^{*} M$ induces a symplectic structure on $\Lambda_{\mathcal{H}}$ making it into a conic, symplectic manifold. Using the representation of $\Lambda_{\mathcal{H}}$ as $M \times \mathbb{R}^{+}$, the induced symplectic form can be written

$$
d(\lambda \theta)=(d \lambda \wedge \theta+\lambda d \theta) .
$$

This symplectic manifold is called the symplectization of the contact manifold, and the previous construction is called the contactization of a conic, symplectic manifold.

In symplectic geometry, the first fundamental theorem was proved by Darboux, and it was, in some ways a little bit discouraging. Darboux's theorem states that a symplectic manifold has no local invariants. The technical statement is the following.

Theorem 2. If $(Y, \omega)$ is symplectic, given $y \in Y$ there exists an open neighborhood $U_{y}$ of $y$ and a diffeomorphism $\psi_{y}: U_{y} \rightarrow \mathbb{R}^{2 n}$ such that

$$
\psi_{y}^{*}\left(\sum d x_{i} \wedge d \xi_{i}\right)=\left.\omega\right|_{U_{y}} .
$$

It says that from the point of view of symplectic geometry, a neighborhood of any point in a symplectic manifold looks exactly like a neighborhood of a point in $\mathbb{R}^{2 n}$ with its standard symplectic structure. There is an analogous statement for contact manifolds.

Theorem 3. Let $M$ be a contact manifold with contact form $\theta$, for all $x \in M$, there exists an open neighborhood $U_{x} \ni x$ and a diffeomorphism $\psi_{x}: U_{x} \rightarrow \mathbb{R}^{2 n+1}$ such that

$$
\psi_{x}^{*}\left(\theta_{0}\right)=\theta
$$

where $\theta_{0}$ is given by (10).

Darboux's theorem says that, even after a contact form is selected, a contact manifold has no local invariants. A reference for this material is [1].

Now we get to the second class of contact manifolds, those arising in complex geometry. On $\mathbb{R}^{2 n}$ we define the standard complex structure by choosing the sub-bundle $T^{1,0} \mathbb{C}^{n} \subset$ $T \mathbb{R}^{2 n} \otimes \mathbb{C}$ spanned at each point by $\left\{\partial_{z_{1}}, \ldots, \partial_{z_{n}}\right\}$ where $\partial_{z_{j}}=\frac{1}{2}\left(\partial_{x_{j}}-i \partial_{y_{j}}\right)$. We also define

$$
T^{0,1} \mathbb{C}^{n}=\overline{T^{1,0} \mathbb{C}^{n}}
$$

The complex structure defines a canonical differential operator on functions:

$$
\bar{\partial} f=\left.d f\right|_{T^{0,1} \mathbb{C}^{n}}=\sum_{j=1}^{n} \partial_{\bar{z}_{j}} f d \bar{z}_{j} .
$$

In one complex dimension a hypersurface, that is a real curve, does not have any holomorphic geometry associated with it because any curve in the plane can be mapped, as the boundary value of a holomorphic map, onto a segment of the real line. Once again, in some sense, there are no local invariants. 
If $n>1$ then the complex structure defines a structure on a hypersurface, called a CR-structure. Let $\rho$ be a smooth function on $\mathbb{C}^{n}$ such that $d \rho \neq 0$ where $\rho=0$ and $M=\left\{\rho^{-1}(0)\right\}$, be a smooth connected hypersurface in $\mathbb{C}^{n}$. We define the "complex tangent space" of $M$ to be

$$
T^{0,1} M=T^{0,1} \mathbb{C}^{n} \uparrow_{M} \cap(T M \otimes \mathbb{C}) .
$$

If $\bar{Z}$ is an element of $T_{p}^{0,1} \mathbb{C}^{n}$ for $p \in M$ then $\bar{Z} \in T_{p}^{0,1} M$ if both $\operatorname{Re} \bar{Z}$ and $\operatorname{Im} \bar{Z}$ are tangent to $M$.

The differential, $d \rho$ restricted to $M$ vanishes since $\rho$ is constant on $M$. We can write $d \rho=\partial \rho+\bar{\partial} \rho$. Hence

$$
\theta:=\frac{1}{i} \partial \rho\lceil M
$$

is a real, non-vanishing 1-form on $M$ and, $\operatorname{ker} \theta \subset T M$ is a real hyperplane bundle. Clearly $T^{0,1} M \oplus T^{1,0} M \subset \operatorname{ker} \theta \otimes \mathbb{C}$. When does $\operatorname{ker} \theta$ defines a contact structure? Writing $d \theta$ in complex coordinates we see that:

$$
d \theta=\frac{1}{i}(d \bar{\partial} \rho) \uparrow_{M}=\frac{1}{i} \sum \frac{\partial^{2} \rho}{\partial z_{j} \partial \bar{z}_{j}} d z_{j} \wedge d \bar{z}_{j} \uparrow_{M}
$$

The condition we need is that $\theta \wedge[d \theta]^{n-1} \neq 0$. There is a neat way to express this condition in terms of complex geometry. The two form $i d \theta$ defines an hermitian form, called the Levi form: $\mathcal{L}^{M}: T^{1,0} M \times T^{1,0} M \rightarrow \mathbb{C}$,

$$
\mathcal{L}^{M}(Z, W)=i d \theta(Z, \bar{W}) .
$$

Proposition 3. The hyperplane bundle, $\operatorname{ker} \theta$ is a contact field if and only if the Levi form is non-degenerate.

Exercise 5. Prove this statement

In complex analysis it is customary to use a negative defining function. Thus $M$ is considered to be the boundary of the set where $X=\{z \mid \rho(z)<0\}$. If the Levi form defined by this choice of $\rho$ is strictly positive (negative) definite, we say that $M$ is the strictly pseudoconvex (pseudoconcave) boundary of $X$. Fixing a sign for the defining function determines a co-orientation for the contact field on $M$. A simple example is provided by the unit ball. In this case $\rho=|z|^{2}-1$, the 1 -form $i \partial \rho \uparrow_{S^{1}}$ is given by

$$
\theta=\sum_{j=1}^{n}\left[x_{j} d y_{j}-y_{j} d x_{j}\right]
$$

and

$$
d \theta=2 \sum_{j=1}^{n} d x_{j} \wedge d y_{j}
$$

A calculation shows that $\theta \wedge[d \theta]^{(n-1)}$ is a positive multiple of the standard volume form on the sphere. The unit sphere can also be thought of as sitting in $\mathbb{P}^{n}$. The exterior of the unit sphere is the set $\rho>0$, so $-\rho$ is a defining function for the exterior of the unit sphere in $\mathbb{P}^{n}$. With the respect to this defining function the Levi form of $S^{2 n-1}$ is negative definite, hence the unit sphere is the strictly pseudoconcave boundary of the exterior of the unit ball.

Thus far we have defined the CR-structure induced on a hypersurface in $\mathbb{C}^{n}$. This is a local construction which can therefore be used to define a CR-structure on a real hypersurface in any complex manifold. We extract the main properties of hypersurfaces in complex manifolds to give a definition for an abstract CR-manifold which does not require an embedding into an ambient complex space. 
Definition 4. A CR-manifold $M$ is an odd-dimensional manifold with a sub-bundle

$$
T^{0,1} M \subset T M \otimes \mathbb{C}
$$

such that

Dimension Fiber dimension of $T^{0,1} M=(\operatorname{dim} M-1) / 2$.

Non-degeneracy $\quad T^{1,0} M \cap T^{0,1} M=\{0\}$.

Integrability If $\bar{Z}, \bar{W} \in \mathcal{C}^{\infty}\left(M, T^{0,1} M\right)$ then, so is $[\bar{Z}, \bar{W}]$.

It is easy to see that these conditions are satisfied if $T^{0,1} M$ is defined by (13) for $M$ a hypersurface in a complex manifold.

In general, $T^{1,0} M \oplus T^{0,1} M=\mathcal{H} \otimes \mathbb{C}$ where $\mathcal{H}=\operatorname{ker} \theta$. As before $i d \theta$ defines the Levi form:

$$
\mathcal{L}^{M}: T^{1,0} M \times T^{1,0} M \rightarrow \mathbb{C} .
$$

A co-orientation for $\mathcal{H}$ fixes the notion of a positive contact form and therefore a sign for $\mathcal{L}^{M}$ (if it is definite). A diffeomorphism $\phi: M \rightarrow N$ between CR-manifolds is a CR-map if

$$
\phi_{*}: T^{0,1} M=T^{0,1} N .
$$

A map $\phi: M \rightarrow \mathbb{C}^{n}$ is a $C R$-embedding if

$$
\phi_{*} T^{0,1} M=T^{0,1} \phi(M)
$$

where $T^{0,1} \phi(M)$ is the CR-structure induced on the hypersurface on $\phi(M) \subset \mathbb{C}^{n}$. More generally we define a CR-embedding of a CR-manifold into $\mathbb{C}^{N}$ for any $N$ as an embed$\operatorname{ding} \phi: M \rightarrow \mathbb{C}^{N}$ such that

$$
\phi_{*} T^{1,0} M=T^{1,0} \phi(M):=T^{1,0} \mathbb{C}^{N} \uparrow_{\phi(M)} \cap T \phi(M) \otimes \mathbb{C} .
$$

In general, for $M$ a real submanifold of $\mathbb{C}^{N}$ of co-dimension greater than $1, T^{1,0} \mathbb{C}^{N} \uparrow_{M}$ $\cap T M \otimes \mathbb{C}$ is not a sub-bundle as the fiber dimension varies from point to point.

Unlike the case of symplectic and contact manifolds, CR-manifolds do have local invariants. The Levi form defines the simplest invariant. An hermitian form $h$ has a single invariant, its signature. The signature is a triple of integers $\left(n_{-}, n_{+}, n_{0}\right)$ where $n=$ $n_{-}+n_{+}+n_{0} ; n_{+}$is the maximum dimension of the subspaces on which $h$ is positive definite and $n_{-}$is the maximum dimension of the subspaces on which $h$ is negative definite. For each point $p \in M$ the signature of $\mathcal{L}_{p}^{M}$ is a CR-invariant.

Proposition 4. The signature of the Levi form is a CR-invariant. That is: if $M$ and $N$ are CR-manifolds and $\phi: M \rightarrow N$ is a co-orientation preserving CR-map then the signature of $\mathcal{L}_{p}^{M}$ equals the signature of $\mathcal{L}_{\phi(p)}^{N}$.

Exercise 6. Prove the proposition.

Exercise 7. Suppose that $M^{2 n-1}$ is a compact hypersurface in $\mathbb{C}^{n}$. If $\mathcal{L}^{M}$ is everywhere non-degenerate, then $M$ is strictly pseudoconvex.

Exercise 8. Let $B_{1}$ denote the unit ball in $\mathbb{C}^{n}$ and let $U$ be a neighborhood of a point on the boundary. Suppose that $\phi: U \cap B_{1} \rightarrow \mathbb{C}^{n}$ is an injective, holomorphic map, smooth up to $U \cap \overline{B_{1}}$. If $\phi\left(U \cap S^{2 n-1}\right) \subset S^{2 n-1}$ then $\phi\left(U \cap B_{1} \subset B_{1}\right)$.

If a real hypersurface in $\mathbb{C}^{n}$ is transverse to the radial vector field then restricting the 1 -form

$$
\alpha=\sum_{j=1}^{n}\left[x_{j} d y_{j}-y_{j} d x_{j}\right]
$$


to the hypersurface also defines a contact structure. This contact structure is generally different from the contact structure induced on a strictly pseudoconvex hypersurface from the complex structure of $\mathbb{C}^{n}$. We finish this section by considering the intersection of the two types of examples. Let $X$ be a real analytic manifold and let $\mathcal{X}$ be the complexification of $X$. Then $X$ embeds into $\mathcal{X}$ as a totally real submanifold. The tubular neighborhood theorem implies that there are diffeomorphisms from a neighborhood of $X$ in $\mathcal{X}$ to a neighborhood of the zero section of $T^{*} X$.

$$
\phi: \mathcal{X} \rightarrow T^{*} X
$$

which reduce to the identity on $X$. One can fix this diffeomorphism so that the induced complex structure on the image of $\phi$ satisfies certain properties. Choose a real analytic Riemannian metric $g$ on $X$. This defines a function $\rho_{g}(\xi)=\|\xi\|^{2}$ on $T^{*} X$. The map, $\phi$ can be chosen so that

(a) $\phi: X \rightarrow X$ is the identity.

(b) With the complex structure induced on the image, $T^{0,1} \phi(\mathcal{X})=\phi_{*} T^{0,1} \mathcal{X}$, we require that $\mathcal{I}:(x, \xi) \rightarrow(x,-\xi)$ is an anti-holomorphic map.

(c) $\operatorname{Im} \bar{\partial} \rho_{g}=\alpha=\sum_{i=1}^{n} \xi_{i} d x_{i}$.

(d) $\left(\partial \bar{\partial} \sqrt{\rho_{g}}\right)^{n}=0$, away from $\rho_{g}^{-1}(0)$.

Let $\mathcal{X}_{\epsilon}=\left\{\rho_{g} \leq \epsilon^{2}\right\}$. It follows from the second item above that the contact structure induced on $b \mathcal{X}_{\epsilon}$ by the complex structure agrees with the contact structure defined on $b \mathcal{X}_{\epsilon}$ by the symplectic structure on $T^{*} X$. The subsets $\mathcal{X}_{\epsilon}$ are called Grauert tubes. For more on this construction see [29] or [17].

\section{CR-FUnCTIONS AND A GENERALIZATION OF TOEPLitz OPERATORS}

On a CR-manifold there is a canonical differential operator, analogous to the $\bar{\partial}$-operator defined by

$$
\bar{\partial}_{b} f:=d f \uparrow_{T^{0,1} M} .
$$

If $\Phi=\left(\phi_{1}, \ldots, \phi_{N}\right)$ is an embedding of $M$ into $\mathbb{C}^{N}$ then, $\Phi$ is a CR-embedding if and only if $\bar{\partial}_{b} \phi_{i}=0$ for all $i=1, \ldots, N$. It follows from the Leibniz formula that the kernel of $\bar{\partial}_{b}$ is an algebra. If $M$ is the boundary of a domain $X$ in $\mathbb{C}^{n}$ then the ker $\bar{\partial}_{b}$ consists of the restrictions of holomorphic functions in $X$ to $M$. More generally if $M$ is an abstract, compact, strictly pseudoconvex CR-manifold which is the boundary of $X$, a compact, complex manifold with boundary, then the ker $\bar{\partial}_{b}$ again consists of the restrictions to $M$ of holomorphic functions on $X$. If $\operatorname{dim} M \geq 5$ then such a compact, complex manifold with boundary always exists. Moreover the induced co-orientation of the contact field agrees with the given co-orientation. If $\operatorname{dim} M=3$ then the existence of such a complex manifold is a very subtle question. Generically such a manifold does not exist, see [11].

A Stein space is a complex space with a very large family of holomorphic functions. Indeed it follows from results of Remmert that any Stein space admits a proper holomorphic embedding into $\mathbb{C}^{N}$, for some $N$. Grauert showed that a compact, complex manifold with a strictly pseudoconvex boundary is a "proper modification" of a Stein space. From work of Heunemann and Ohsawa it follows that a strictly pseudoconvex manifold with boundary $X$ can be augmented along its boundary so that $X \subset \tilde{X}$ is a relatively compact subset in a proper modification of a Stein space and $M$ is therefore a proper hypersurface in $\tilde{X}$.

If a function $f \in \operatorname{ker} \bar{\partial}$ on $\tilde{X}$ then $f \uparrow_{M}$ is in ker $\bar{\partial}_{b}$. Restricting a proper holomorphic embedding of $\widetilde{X}$ into $\mathbb{C}^{N}$ to $M$ we obtain a CR-embedding of $M$ into $\mathbb{C}^{N}$. In this case the algebra of CR-functions is large, containing the closure of the algebra generated by the coordinate functions of the embedding. Using work of Boutet de Monvel, Kohn and 
Harvey and Lawson, one can show that a compact, strictly pseudoconvex CR-manifold has a CR-embedding into $\mathbb{C}^{N}$, for some $N$ if and only if it is the boundary of a strictly pseudoconvex, complex manifold with boundary, see [6], [22] and [18]. We call such a CR-manifold embeddable.

We can generalize the construction of a Toeplitz operator defined earlier on the boundary of the unit disk to an embeddable, strictly pseudoconvex CR-manifold. Suppose that $M$ is such a manifold. The ker $\bar{\partial}_{b}$ is infinite dimensional, containing enough functions to separate points on $M$. Choose a volume form on $M$, and let $H^{2}(M)$ denote the $L^{2}$ closure of ker $\bar{\partial}_{b}$ and $S$ denote the orthogonal projection

$$
S: L^{2}(M) \rightarrow H^{2}(M) .
$$

This is called the Szegó projector. The Szegó projector is not canonically defined as it requires the choice of a volume form. The image of $S$ is canonical, but the null space is not. A complex valued function $a$ on $M$ defines a Toeplitz operator by setting

$$
T_{a}=S a S: H^{2}(M) \rightarrow H^{2}(M) .
$$

This class of operators can easily be enlarged, we can allow $A$ to be a pseudodifferential operator and then define $T_{A}$ by the same formula: $T_{A}=S A S$. As we shall see this only slightly expands the class of operators that one obtains.

Understanding the Szegó projector is the key to understanding and further generalizing Toeplitz operators. The Szegó projector cannot be a standard pseudodifferential operator. This is a consequence of the symbolic properties of such operators. It turns out that the Szegó projector does belong to a slightly exotic calculus of pseudodifferential operators called the Heisenberg calculus. To prepare for our discussion of it we now review the basic facts about standard pseudodifferential operators.

\section{PSEUdODIFFERENTIAL OPERATORS, SYMBOLS AND THE RADIAL COMPACTIFICATION OF THE COTANGENT BUNDLE}

We begin our study of operators in a very general setting. Given a linear operator $A: \mathcal{C}_{\mathrm{c}}^{\infty}\left(\mathbb{R}^{n}\right) \rightarrow \mathcal{C}^{\infty}\left(\mathbb{R}^{n}\right)$, one would like to represent it as integration against a kernel. In a certain sense, this is always possible.

Theorem 4 (Schwartz kernel theorem). There exists a unique element

$$
k_{A} \in \mathcal{C}^{-\infty}\left(\mathbb{R}^{n} \times \mathbb{R}^{n}\right)
$$

such that

$$
A f=\int k_{A} f
$$

in the weak sense, i.e.

$$
<A f, g>=<k_{A}, f(x) \otimes g(y)>
$$

for every $f, g \in \mathcal{C}_{\mathrm{c}}^{\infty}\left(\mathbb{R}^{n}\right)$

Using the variables $x, x-y$ we can represent this as a family of convolution operators:

$$
A f(x)=\int k_{A}(x, x-y) f(y) d y
$$

We make the following assumptions on the kernel $k_{A}$ :

(a) $k_{A}(x, z)$ a smooth function on $\mathbb{R}_{x}^{n}$ with values in tempered distributions on $\mathbb{R}_{z}^{n}$.

(b) $k_{A}(x, z)$ is in $\mathcal{C}^{\infty}\left(\mathbb{R}^{n} \times\left(\mathbb{R}^{n} \backslash\{0\}\right)\right)$ - the only singularities are along the diagonal, i.e. where $z=0$. 
(c) In virtue of our first assumption for each fixed $x, k_{A}(x, \cdot)$ has a Fourier transform. Let $a(x, \xi)$ be the partial Fourier transform of the kernel, that is

$$
a(x, \xi)=<k_{A}(x, z), e^{-i \xi z}>\in \mathcal{C}^{\infty}\left(\mathbb{R}^{n} ; \mathcal{S}^{\prime}\left(\mathbb{R}^{n}\right)\right) .
$$

If we assume that the support of $k_{A}$ in $z$ is compact then $a(x, \xi) \in \mathcal{C}^{\infty}\left(\mathbb{R}^{n} \times \mathbb{R}^{n}\right)$. Let $\varphi \in \mathcal{C}_{c}^{\infty}(\mathbb{R})$ with $\varphi(t)=1$ for $|t|<1$ and $\varphi(t)=0$ for $|t|>2$ then we can write

$$
k_{A}(x, z)=\varphi(\|z\|) k_{A}(x, z)+(1-\varphi(\|z\|)) k_{A}(x, z) .
$$

The first term has compact support in $z$ whereas the second term belong to $\mathcal{C} \infty\left(\mathbb{R}^{n} \times \mathbb{R}^{n}\right)$. Since we are more interested in the singular term we assume, provisionally that $k_{A}(x, z)$ has compact support in $z$. Indeed, in our applications we work on compact manifolds and then we can always arrange this by using a partition of unity.

With these assumptions $A$ has a representation as a Fourier integral:

$$
A f=\int a(x, \xi) e^{i \xi x} \hat{f}(\xi) d \xi .
$$

Here, $a(x, \xi)$ is called the symbol of the linear operator $A$. For example, let us consider a differential operator

$$
A f(x)=\sum_{\substack{|\alpha| \leq k \\ \alpha=\left(\alpha_{1}, \cdots, \alpha_{n}\right)}} a_{\alpha}(x) D^{\alpha} f(x),
$$

where $D=-i\left(\partial_{x_{1}}, \ldots, \partial_{x_{n}}\right)$ and the $\alpha$ are multi-indices. One can check that the symbol of $A$ is

$$
a(x, \xi)=\sum_{|\alpha| \leq k} a_{\alpha}(x) \xi^{\alpha} .
$$

The raison d'etre for pseudodifferential operators is to translate analytic properties of linear operators into algebraic properties of the symbol and vice versa. It might appear that with all these assumptions we would have reduced our consideration to a small family of operators. In fact, this is still far too general a class of operators to have a useful theory. To obtain analyzable classes of operators we need to place further restrictions on the symbols. Kohn and Nirenberg introduced the class of symbols that bear their name, in part for the purpose of studying the $\bar{\partial}$-Neumann problem, see [24, 23]. These are also called classical or polyhomogeneous, step-1 symbols.

A smooth function $a(x, \xi)$ is a classical symbol if it has an asymptotic expansion:

$$
a(x, \xi) \sim \sum_{j=-\infty}^{m} a_{j}(x, \xi) \text { for large } \xi
$$

where $a_{j}(x, \lambda \xi)=\lambda^{j} a_{j}(x, \xi)$ and $a_{j}$ are smooth in $\mathbb{R}^{n} \times\left(\mathbb{R}^{n} \backslash\{0\}\right)$. Here $\sim$ means that for arbitrary multi-indices $\alpha, \beta$ and $N \leq m$ there exist constants $\left\{C_{\alpha \beta}^{N}\right\}$ such that

$$
\left|D_{x}^{\alpha} D_{\xi}^{\beta}\left[a(x, \xi)-\sum_{j=-N}^{m} a_{j}(x, \xi)\right]\right| \leq C_{\alpha \beta}(1+|\xi|)^{m-N-|\beta|-1} \text { for }|\xi|>1 .
$$

Note that differentiating with respect to $\xi$ improves the order of approximation. The number $m$ is called the order of the symbol.

Definition 5. We denote the set of classical symbols of order $m$ by $S_{\mathrm{KN}}^{m}\left(\mathbb{R}^{n}\right)$. The first term in $(15), a_{m}(x, \xi)$, is called the principal symbol.

The symbol $a(x, \xi)$ has an expansion at infinity in the $\xi$-parameter in terms of homogeneous functions. This can be interpreted as a Taylor expansion for the function $a(x, \xi)$ 
along the boundary of a compactification of $\mathbb{R}^{n} \times \mathbb{R}^{n}$ in the $\xi$-directions. Indeed a classical symbol of order zero turns out to be nothing more than a smooth function on this compactified space.

We identify $\mathbb{R}^{n}$ with the affine hyperplane $x_{n+1}=1$ in $\mathbb{R}^{n+1}$. Projecting this hyperplane onto the unit sphere in $\mathbb{R}^{n+1}$ carries $\mathbb{R}^{n}$ to the open upper hemisphere, $S_{+}^{n}$. We can therefore compactify $\mathbb{R}^{n}$ by adding the equator of $S_{+}^{n}$. This space, denoted by ${ }^{\operatorname{rad}} \overline{\mathbb{R}}^{n}$, is called the radial compactification of $\mathbb{R}^{n}$. Polar spherical coordinates are defined by:

$$
\omega=\frac{\xi}{|\xi|} \text { and } \rho=\frac{1}{|\xi|}
$$

The parameters $(\omega, \rho)$ define a local coordinates at infinity. A function on ${ }^{\operatorname{rad}} \overline{\mathbb{R}}^{n}$ is smooth near to infinity if and only if is a smooth function of the variables $(\omega, \rho)$. The boundary of $\operatorname{rad} \overline{\mathbb{R}}^{n}$ is the set $\{\rho=0\}$.

We now replace $\mathbb{R}^{n} \times \mathbb{R}^{n}$ by $\mathbb{R}^{n} \times \operatorname{rad} \overline{\mathbb{R}}^{n}$. Suppose that $a$ is a symbol of order zero. Rewriting the asymptotic expansion we have that

$$
a(x, \xi) \sim \sum_{j=-\infty}^{0} a_{j}(x, \xi)=\sum_{j=-\infty}^{0} a_{j}\left(x, \frac{\omega}{\rho}\right)
$$

Recalling that $a_{j}$ is homogeneous of degree $j$, we can rewrite this as

$$
a(x, \xi) \sim \sum_{j=0}^{\infty} \rho^{j} a_{-j}(x, \omega) .
$$

The conditions in (16) imply that $a$ extends smoothly to $\mathbb{R}^{n} \times{ }^{\operatorname{rad}} \overline{\mathbb{R}}^{n}$ and this is the Taylor expansion of the extended function along $\mathbb{R}^{n} \times b^{\text {rad }} \overline{\mathbb{R}}^{n}$. We can therefore identify $S_{\mathrm{KN}}^{m}\left(\mathbb{R}^{n}\right)$ with $\rho^{-m} \mathcal{C}^{\infty}\left(\mathbb{R}^{n} \times{ }^{\operatorname{rad}} \overline{\mathbb{R}}^{n}\right)$. So, up to multiplying by $\rho^{m}$ classical symbols are simply smooth functions on $\mathbb{R}^{n} \times \operatorname{rad} \overline{\mathbb{R}}^{n}$. We have shown that

Proposition 5. The symbol $a(x, \xi) \in S_{\mathrm{KN}}^{m}\left(\mathbb{R}^{n}\right)$ if and only if $\rho^{m} a(x, \xi)$ has a smooth extension to $\mathbb{R}^{n} \times \operatorname{rad} \overline{\mathbb{R}}^{n}$.

At the root of this discussion is the dilation structure on $\mathbb{R}^{n}$. The positive reals, $\mathbb{R}_{+}$acts on $\mathbb{R}^{n}$ by $M_{\lambda}(\xi)=\lambda \xi$. A function is homogeneous of degree $j$ precisely when

$$
M_{\lambda}^{*} f=\lambda^{j} f .
$$

Thus it is the linear dilation structure of $\mathbb{R}^{n}$ which defines the smooth functions on the $\operatorname{rad} \overline{\mathbb{R}}^{n}$.

Now that we have defined symbols, we turn to the operators they define. A symbol $a \in S_{\mathrm{KN}}^{m}$ defines an operator $a(X, D)$ by

$$
a(X, D) f=\frac{1}{[2 \pi]^{n}} \int_{\mathbb{R}^{n}} a(x, \xi) e^{i \xi x} \hat{f}(\xi) d \xi .
$$

This is called the left quantization; the function $a$ is called the symbol of the operator $a(X, D)$.. An operator with such a representation belongs to the Kohn-Nirenberg class of pseudodifferential operators of order $m$, which we denoted by $\Psi_{\mathrm{KN}}^{m}\left(\mathbb{R}^{n}\right)$. If $A \in \Psi_{\mathrm{KN}}^{m}\left(\mathbb{R}^{n}\right)$ then the order $m$ term in the asymptotic expansion of its symbol is called the principal symbol. It is denoted by $\sigma_{m}(A)$.

In functional analysis a basic tool for studying an operator is the functional calculus. This is especially powerful for self-adjoint operators. If two self adjoint operators commute then they can be simultaneously diagonalized and again the functional calculus is a very power tool for studying the relationships between the two operators. An underlying 
motivation for introducing classical pseudodifferential operators is that all such operators are simultaneously approximately diagonalized by the Fourier transform. Moreover we can use this representation to construct an approximate functional calculus for the entire algebra.

Recall the definition of $L^{2}$-Sobolev spaces, $H^{s}\left(\mathbb{R}^{n}\right)$ for $s \in \mathbb{R}$.

Definition 6. A tempered distribution $u$ belongs to $H^{s}\left(\mathbb{R}^{n}\right)$ if its Fourier transform $\hat{u}$ is a measurable function such that

$$
\|u\|_{H^{s}}^{2}=\int|\hat{u}(\xi)|^{2}\left(1+|\xi|^{2}\right)^{s} d \xi
$$

is finite.

Pseudodifferential operators have simple mapping properties on $L^{2}$-Sobolev spaces. The following theorem summarizes the basic properties of the Kohn-Nirenberg class of pseudodifferential operators.

Theorem 5. Suppose that $A$ and $B$ are Kohn-Nirenberg pseudodifferential operators.

(a) [Symbol filtered algebra] If $A \in \Psi_{\mathrm{KN}}^{m}\left(\mathbb{R}^{n}\right)$ and $B \in \Psi_{\mathrm{KN}}^{m^{\prime}}\left(\mathbb{R}^{n}\right)$, then $A \circ B \in$ $\Psi_{\mathrm{KN}}^{m+m^{\prime}}\left(\mathbb{R}^{n}\right)$.

(b) [Composition formula for principal symbols] Let $\sigma_{m}(A), \sigma_{m^{\prime}}(B)$ be the principal symbols of $A$ and $B$ then the principal symbol of the composite is given by the pointwise product:

$$
\sigma_{n+m}(A \circ B)(x, \xi)=\sigma_{n}(A)(x, \xi) \cdot \sigma_{m}(B)(x, \xi) .
$$

This is simultaneous diagonalization, to leading order.

(c) [Mapping properties] If $A \in \Psi_{\mathrm{KN}}^{m}\left(\mathbb{R}^{n}\right)$, then for all $s \in \mathbb{R}$ we have

$$
A: H^{s}\left(\mathbb{R}^{n}\right) \rightarrow H^{s-m}\left(\mathbb{R}^{n}\right) .
$$

This states that if $A \in \Psi_{\mathrm{KN}}^{m}\left(\mathbb{R}^{n}\right), m$ a natural number then its Sobolev space mapping properties are the same as those of a differential operator of order $m$.

Let

$$
\Psi_{\mathrm{KN}}^{-\infty}\left(\mathbb{R}^{n}\right)=\bigcap_{j=-\infty}^{\infty} \Psi_{\mathrm{KN}}^{j}\left(\mathbb{R}^{n}\right) .
$$

An operator in $\Psi_{\mathrm{KN}}^{-\infty}\left(\mathbb{R}^{n}\right)$ is called a smoothing operator, its symbol, $a(x, \xi)$ belongs to $\mathcal{C}^{\infty}\left(\mathbb{R}_{x}^{n}, \mathcal{S}\left(\mathbb{R}_{\xi}^{n}\right)\right)$. If $A$ is a smoothing operator then

$$
A: \mathcal{C}_{\mathrm{c}}^{-\infty} \rightarrow \mathcal{C}^{\infty}
$$

In the context of pseudodifferential operators, the smoothing operators are considered to be negligible. For example a pseudodifferential operator $A$ is considered "invertible" if there is another pseudodifferential operator $B$ such that both $A B-\operatorname{Id}$ and $B A-\operatorname{Id}$ are smoothing operators. This is, of course not the same thing as being invertible, it is a close as one can come working entirely within the confines of pseudodifferential operators.

Definition 7. A pseudodifferential operator $A$ of order $m$ is elliptic if $\sigma_{m}(A)$ is nonvanishing.

It is a consequence of the formula for the symbol of a composition that an elliptic pseudodifferential operator is invertible up to a smoothing error.

Proposition 6. If $A$ is a pseudodifferential operator of order $m$ then there exists a pseudodifferential operator $B$ of order $-m$ such that $A \circ B-\operatorname{Id}$ and $B \circ A-\operatorname{Id}$ are smoothing operators if and only if $A$ is elliptic. 
To define pseudodifferential operators on a compact manifold, one needs to check that

$$
\left(\psi^{-1}\right)^{*} A \psi^{*} \in \Psi_{\mathrm{KN}}^{m}\left(\mathbb{R}^{n}\right) \text { provided } A \in \Psi_{\mathrm{KN}}^{m}\left(\mathbb{R}^{n}\right)
$$

for any $\psi \in \operatorname{Diff}\left(\mathbb{R}^{n}\right)$ which is the identity outside of a compact set. This implies that under changes of coordinate, the operator may be changed, but the class of operators, including its order filtration is not changed. A proof of this fact along with all the other results reviewed here can be found in [21].

Definition 8. Let $X$ be a manifold. An operator $A: \mathcal{C}_{\mathrm{c}}^{\infty}(X) \rightarrow \mathcal{C}^{-\infty}(X)$ is called a pseudodifferential operator on $X$ of order $m$ if the following conditions are satisfied.

(a) Given $\varphi, \psi \in \mathcal{C}_{\mathrm{c}}^{\infty}(X)$ such that $\operatorname{supp} \varphi \cap \operatorname{supp} \psi=\emptyset$, then the operator $\varphi A \psi$ is a smoothing operator;

$$
\varphi A \psi: \mathcal{C}^{-\infty}(X) \rightarrow \mathcal{C}^{\infty}(X)
$$

(b) If $\operatorname{supp} \varphi$ is contained in a coordinate chart $U$, then with respect to any choice of local coordinates on $U$ the operator $\varphi A \varphi \in \Psi_{\mathrm{KN}}^{m}\left(\mathbb{R}^{n}\right)$.

Let $T^{*} X$ denote the cotangent bundle of $X$. The fibers of $X$ are real vector spaces and therefore have a canonical $\mathbb{R}_{+}$-action. One of the most useful properties of pseudodifferential operators is the fact that if $A$ is a pseudodifferential operator of order $m$, then its principal symbol $\sigma_{m}(A)$ is a function on $T^{*} X \backslash\{0\}$, homogeneous with respect to the canonical $\mathbb{R}_{+}$-action on the fibers. If $x$ are local coordinates for $X$ with dual coordinates $\xi$ on the fibers of $T^{*} X$ then

$$
\sigma_{m}(A)(x, \lambda \xi)=\lambda^{m} \sigma_{m}(A)(x, \xi) .
$$

That the principal symbol is a function is by no means obvious. One needs to examine the transformation law for the symbol of $A$ under a change of local coordinates. In general this dependence is very complicated. It so happens that the leading term in the asymptotic expansion of the symbol transforms as a function on the co-tangent space itself. For the details of this calculation see [21].

Because the principal symbol is well defined as a function we can transfer the notion of ellipticity to a manifold.

Definition 9. A pseudodifferential operator $A$ of order $m$ defined on $X$ is elliptic if $\sigma_{m}(A)$ is nowhere vanishing on the co-sphere bundle $S^{*} X$.

An elliptic pseudodifferential operator on a compact manifold is invertible up to a compact error. Suppose that the principal symbol $\sigma_{m}(A)(x, \xi)$ does not vanish for all $(x, \xi)$ where $\xi \neq 0$. We define a new symbol by setting

$$
b(x, \xi)=\frac{\varphi(|\xi|)}{\sigma_{m}(A)(x, \xi)}, \text { where } \varphi(s)= \begin{cases}0 & \text { if } s<1 \\ 1 & \text { if } s>2 .\end{cases}
$$

If $b(X, D)$ is the quantization of this symbol then it has the following properties:

$$
A \circ b(X, D)-I \in \Psi_{\mathrm{KN}}^{-1}(X), \text { and } b(X, D) \circ A-I \in \Psi_{\mathrm{KN}}^{-1}(X) .
$$

Using the composition formula for pseudodifferential operators, we can actually find an operator $B$ such that

$$
A \circ B-I \in \Psi_{\mathrm{KN}}^{-\infty}(X) \text {, and } B \circ A-I \in \Psi_{\mathrm{KN}}^{-\infty}(X),
$$

where $\sigma_{-m}(B)=b$.

A consequence of this almost invertibility is a criterion for a pseudodifferential operator to be Fredholm. 
Proposition 7. If $A \in \Psi_{\mathrm{KN}}^{m}(X)$ is elliptic then $A$ is a Fredholm operator as a map $A$ : $H^{s}(X) \rightarrow H^{s-m}(X)$ for any $s$.

It is now easy to show that, on a compact manifold of dimension at east 2 , the range of a classical pseudodifferential projection has either finite dimension or finite co-dimension. Let $P$ be an orthogonal projection, acting on functions which is a standard pseudodifferential operator. As it is bounded it must be an operator of order 0 . Since $P^{2}-P=0$ it would follow that

$$
\sigma_{0}(P)\left(\sigma_{0}(P)-1\right) \equiv 0 .
$$

Hence $\sigma_{0}(P)$ is either identically 0 or identically 1 . If $\sigma_{0}(P)$ is zero then $P$ is an operator of order -1 and hence compact. It therefore has a finite dimensional range. If $\sigma_{0}(P)$ is one then $P$ is Fredholm and therefore has a finite co-dimensional ranage. The Szegó projector has both infinite rank and infinite co-rank and therefore cannot be a classical pseudodifferential operator.

Exercise 9. The projector $\pi$ onto $H^{2}\left(S^{1}\right)$ is a classical pseudodifferential operator. Can you explain why this does not contradict the argument in the previous paragraph.

Exercise 10. Show that the Szegó projector has an infinite co-dimensional range. Hint: Consider complex conjugates of holomorphic functions.

\section{PARABOLIC COMPACTIFICATIONS FOR CONTACT MANIFOLDS}

Now we let $X$ be a contact manifold. On a contact manifold it is possible to define a different $\mathbb{R}_{+}$-action on the co-tangent space. This in turn leads to a different compactification of $T^{*} X$ and thereby to a different class of symbols. To define the $\mathbb{R}_{+}$-action we choose a contact form $\theta$. This defines the Reeb vector field $T$ which satisfies

(a) $\theta(T)=0$.

(b) $i_{T} d \theta=0$, i.e. $d \theta(T, W)=0$ for all $W \in T X$.

Using the vector field $T$ we can define a complement for the $\theta$-direction in the fibers of $T^{*} X$. Let $T^{\perp}$ be the set of one forms such that $\eta(T)=0$, i.e.

$$
T^{\perp}=\{\eta \mid \eta(T)=0\}=(\operatorname{ker} \theta)^{*} \subset T^{*} X .
$$

This decomposes $T^{*} X$ as the Whitney sum of two subbundles

$$
T^{*} X=\mathbb{R} \theta \oplus T^{\perp} \text {. }
$$

Which in turn defines two bundle maps by projection onto the subbundles,

$$
\begin{array}{ll}
\pi_{1}: & T^{*} X \rightarrow T^{\perp}, \\
\pi_{2}: & T^{*} X \rightarrow \mathbb{R} \theta .
\end{array}
$$

We define a parabolic action of $\mathbb{R}_{+}$on $T^{*} X$ by letting

$$
\mathcal{M}_{\lambda}(\xi)=\lambda \pi_{1}(\xi)+\lambda^{2} \pi_{2}(\xi) \text { for } \xi \in T_{x}^{*} X
$$

The trajectories look like parabolas with respect to $\left(\theta, T^{\perp}\right)$ coordinates.

Before we consider the compactification of $T^{*} X$ defined by this action, we examine the action in more detail. To understand this action, we use Theorem 3 to introduce Darboux coordinates. These are local coordinates, $(t, x, y)$ on $X$ such that:

$$
\theta=d t+\frac{1}{2}(x \cdot d y-y \cdot d x)
$$


the Reeb vector field is $T=\partial_{t}$. We employ the following notation for co-vectors

$$
\left(\xi_{0}, \xi^{\prime}\right)=\left(\xi_{0}, \xi_{1}, \ldots, \xi_{2 n}\right) \leftrightarrow \xi_{0} d t+\sum_{j=1}^{2} \xi_{j} d x_{j}+\xi_{j+n} d y_{j} .
$$

The projections in these coordinates are given by:

$$
\begin{aligned}
& \pi_{2}\left(\xi_{0}, \xi^{\prime}\right)=\xi_{0} \theta \\
& \pi_{1}\left(\xi_{0}, \xi^{\prime}\right)=\sum_{i=1}^{n}\left(\xi_{i}+\frac{1}{2} y_{i} \xi_{0}\right) d x_{i}+\left(\xi_{i+n}-\frac{1}{2} x_{i} \xi_{0}\right) d y_{i} .
\end{aligned}
$$

The coefficients are linear functions on the cotangent space and therefore the symbols of vector fields,

$$
\begin{aligned}
\xi_{0} & =\sigma\left(\frac{1}{i} \partial_{t}\right), \\
\xi_{j}+\frac{1}{2} y_{j} \xi_{0} & =\sigma\left(\frac{1}{i}\left(\partial_{x_{j}}+\frac{1}{2} y_{j} \partial_{t}\right)\right), \\
\xi_{j+n}-\frac{1}{2} x_{j} \xi_{0} & =\sigma\left(\frac{1}{i}\left(\partial_{y_{j}}-\frac{1}{2} x_{j} \partial_{t}\right)\right) .
\end{aligned}
$$

We denote these vector fields by:

$$
T:=\partial_{t}, \quad X_{j}:=\partial_{x_{j}}+\frac{1}{2} \partial_{t}, \quad Y_{j}:=\partial_{y_{j}}-\frac{1}{2} x_{j} \partial_{t} .
$$

The contact field is locally spanned by $\left\{X_{j}, Y_{j}\right\}$. These vector fields satisfy the following commutation relations:

$$
\begin{aligned}
{\left[X_{i}, T\right] } & =\left[Y_{k}, T\right]=0, \\
{\left[X_{j}, X_{k}\right] } & =\left[Y_{j}, Y_{k}\right]=0, \\
{\left[X_{j}, Y_{k}\right] } & =-\delta_{j k} T .
\end{aligned}
$$

These relations define the Heisenberg Lie algebra. Integrating these vector fields gives the Heisenberg group structure on $\mathbb{R}^{2 n+1}$. The addition is defined by

$$
(t, x, y) \oplus\left(t^{\prime}, x^{\prime}, y^{\prime}\right)=\left(t+t^{\prime}+\frac{1}{2}\left(y^{\prime} \cdot x-x^{\prime} \cdot y\right), x+x^{\prime}, y+y^{\prime}\right) .
$$

The parabolic dilation structure "twists" as the base point moves. By this we mean that there do not exist local coordinates $\left(x_{0}, \ldots, x_{2 n}\right)$ such that, in terms of the linear coordinates, $\left\{\beta_{j}\right\}$ defined on $T^{*} X$ by the local co-frame $\left\{d x_{0}, \ldots, d x_{2 n}\right\}$ the dilation structure takes the form

$$
\mathcal{M}_{\lambda}(x, \beta)=\left(x ; \lambda^{2} \beta_{0}, \lambda \beta_{1}, \ldots, \lambda \beta_{2 n}\right) .
$$

This is a reflection of the non-integrability of the contact field. Instead we use the vector fields $\left\{T, X_{j}, Y_{k}\right\}$ to "flatten" the dilation structure. The symbols of these vector fields $\left\{\sigma_{0}, \ldots, \sigma_{2 n}\right\}$ give local coordinates for $T^{*} X$ which are, in fact linear coordinates on the fibers. However they do not come from a choice of coordinates on the manifold. With $\sigma^{\prime}=\left(\sigma_{1}, \ldots, \sigma_{2 n}\right)$, the parabolic action takes a simple form in these coordinates:

$$
\mathcal{M}_{\lambda}\left(x, \sigma_{0}, \sigma^{\prime}\right)=\left(x, \lambda^{2} \sigma_{0}, \lambda \sigma\right) .
$$

Using the parabolic dilation structure we define a new compactification on $T^{*} X$. Let $g$ denote a Riemannian metric on $T^{*} X$. The smooth hypersurface:

$$
\Sigma=\left\{\xi:\left|\pi_{1}(\xi)\right|_{g}^{4}+\left|\pi_{2}(\xi)\right|_{g}^{2}=1\right\}
$$


is transverse to the orbits of $\mathcal{M}_{\lambda}$. The set underlying the parabolic compactification of $T^{*} X$ is ${ }^{H} \overline{T^{*} X}=T^{*} X \sqcup \Sigma$. That is: we add one point at "infinity" for each trajectory of the dilation structure. The philosophy behind using a dilation structure to define a compactification is that a smooth function in the complement of the zero section which is homogeneous of non-positive, integral degree should have a smooth extension to the compactified space. With this in mind we let

$$
\rho=\left(\sigma_{0}^{2}+\left|\sigma^{\prime}\right|_{g}^{4}\right)^{-\frac{1}{4}} \text { and } y_{0}=\sigma_{0} \rho^{2}, y_{i}=\sigma_{i} \rho, \quad i=1, \ldots, 2 n .
$$

A function near $b^{H} \overline{T^{*} X}$ is smooth if it is a smooth functions of these variables. In other words appropriate subsets of these variables give local coordinates near to $b^{H} \overline{T^{*} X}$. Notice that $\sigma_{0} \rho^{2}$ and $\sigma^{\prime} \rho$ are homogeneous of degree 0 with respect to the parabolic action, and $\rho$ is homogeneous of degree -1 , it is a defining function for the boundary.

This new compactification defines a new classe of symbols:

Definition 10. A function $a \in \mathcal{C}^{\infty}\left(T^{*} X\right)$ defines a Heisenberg symbol of order $m \in \mathbb{R}$ if $\rho^{m} a$ has a smooth extension as a function in $\mathcal{C}^{\infty}\left({ }^{H} \overline{T^{*} X}\right)$. We denote this set of functions by $S_{\mathrm{H}}^{m}(X)$.

From our discussion of classical symbols it is easily seen that a function $a$ defines a Heisenberg symbol of order $m$ if and only if it has an asymptotic expansion

$$
a \sim \rho^{-m} \sum_{j=-\infty}^{0} a_{j}(x, \xi)
$$

where $a_{j}$ is Heisenberg-homogeneous of order $j$,

$$
\mathcal{M}_{\lambda}^{*} a_{j}=\lambda^{j} a_{j} .
$$

\section{The Heisenberg CAlculus}

The introduction of Darboux coordinates identifies a coordinate chart in $X$ with an open set in the Heisenberg group. For the moment we work in the Heisenberg group itself. We can quantize Heisenberg symbols to obtain operators just as before. Instead of formula (17) we use the Weyl quantization formula

$$
a^{W}(X, D) f=\frac{1}{(2 \pi)^{2 n+1}} \iint a\left(\frac{x+y}{2}, \xi\right) e^{i<(x-y), \xi>} f(y) d y d \xi .
$$

We use this formula because Weyl quantization has better invariance properties than the left quantization used above.

Definition 11. An operator $A: \mathcal{C}_{\mathrm{c}}^{\infty}(X) \rightarrow \mathcal{C}^{-\infty}(X)$ is a Heisenberg operator of order $m$ on $X$ if

(a) For any pair $\varphi, \psi \in \mathcal{C}_{\mathrm{c}}^{\infty}(X)$ with disjoint supports the operator $\psi A \varphi$ is a smoothing operator,

(b) If $\varphi \in \mathcal{C}_{\mathrm{c}}^{\infty}(X)$ has support in a coordinate chart then $\varphi A \varphi$ has a representation as in (22) for a symbol $a \in S_{\mathrm{H}}^{m}(X)$.

The set of such operators is denoted by $\Psi_{\mathrm{H}}^{m}(X)$.

The functions $\left\{\sigma_{1}, \ldots, \sigma_{2 n}\right\}$ are homogeneous of order 1 while $\sigma_{0}$ is homogeneous of order 2. This shows that, in the Heisenberg calculus, the vector fields tangent to $\mathcal{H}$,

$$
\left\{X_{1}, \ldots, X_{n}, Y_{1}, \ldots, Y_{n}\right\}
$$

define operators of order 1 while the vector field $T$ defines a operator of order 2 . 
If $A$ is a standard pseudodifferential operator and $\phi$ is a diffeomorphism of $X$ then $\phi^{*} A \phi^{-1 *}$ is again a standard a pseudodifferential operator. If $A$ is a Heisenberg pseudodifferential operator then in general the conjugated operator is not. A diffeomorphism which preserves the contact structure, i.e. $\phi^{*}(\theta)=e^{\lambda} \theta$ is called a contact transformation. If $\phi$ is a contact transformation then $\phi^{*} A \phi^{-1 *}$ is again a Heisenberg operator. The reason for this is quite simple: If $\phi: X \rightarrow X$ is a diffeomorphism then the map $\phi^{*}: T^{*} X \rightarrow T^{*} X$ has a smooth extension to ${ }^{H} \overline{T^{*} X}$ if and only if $\phi$ is a contact transformation. Observe that the symbols of order zero are defined, in a coordinate invariant way, as the set of smooth functions on a compact manifold with boundary.

Exercise 11. Prove that a diffeomorphism $\phi^{*}: T^{*} X \rightarrow T^{*} X$ has a smooth extension to $H \overline{T^{*} X}$ if and only if $\phi$ is a contact transformation.

If $A \in \Psi_{\mathrm{H}}^{m}(X)$ then it has local representations as in (22) where $a \in S_{\mathrm{H}}^{m}(X)$. Such a symbol has an asymptotic expansion in Heisenberg homogeneous terms. It can again be shown that the leading term in the expansion is well defined modulo lower order terms. We call this the Heisenberg principal symbol of $A$ which we denote by $\sigma_{m}^{\mathrm{H}}(A)$.

In the manifold context we interpret the $\xi$-variable as the linear coordinate on the fibers of $T^{*} X$ induced by the local coordinates $x$. To better understand the link between the class of Heisenberg operators and the Heisenberg group we change variables in (22) as follows: For each $x$ define the $(2 n+1) \times(2 n+1)$ matrix

$$
L_{x}=\left(\begin{array}{ccc}
1 & -\frac{\hat{x}}{2} & \frac{\hat{x}}{2} \\
0 & 1 & 0 \\
0 & 0 & 1
\end{array}\right)
$$

where $x=\left(x_{0}, \tilde{x}, \hat{x}\right), y=\left(y_{0}, \tilde{y}, \hat{y}\right)$, and $\tilde{x}, \hat{x}, \tilde{y}, \hat{y} \in \mathbb{R}^{n}$. Observe that $L_{x}^{-1}=L_{-x}$. If $\oplus$ denote the Heisenberg addition operation then

$$
x \oplus y^{-1}=L_{x}(x-y) .
$$

Now formula (22) reads

$$
\begin{aligned}
a^{W}(X, D) f & =\iint a\left(\frac{x+y}{2}, \xi\right) e^{\left.i<L_{-x}\left(x \oplus y^{-1}\right), \xi>\right)} f(y) d y d \xi \\
& =\iint \mathfrak{a}\left(\frac{x+y}{2}, \sigma\right) e^{i<x \oplus y^{-1}, \sigma>} f(y) d y d \sigma
\end{aligned}
$$

where $L_{-x}^{t} \xi=\sigma$ and $\mathfrak{a}(x, \sigma)=a\left(x, L_{x}^{t} \sigma\right)$. A calculation shows that if $x$ is a local Darboux coordinate then the functions, $\left(\sigma_{0}, \sigma_{1}, \ldots, \sigma_{2 n}\right)$ agree with the symbols defined earlier in (18). The last integral expresses $a^{W}(X, D)$ as a smooth family of convolution operators on the Heisenberg group. In these coordinates on $T^{*} X$ the principal symbol satisfies

$$
\sigma_{m}^{\mathrm{H}}(A)\left(x, \lambda^{2} \sigma_{0}, \lambda \sigma^{\prime}\right)=\lambda^{m} \sigma_{m}^{\mathrm{H}}(A)\left(x, \sigma_{0}, \sigma^{\prime}\right) .
$$

The composition formula for symbols in the Heisenberg calculus is quite different from that in the classical calculus. In order to work out this formula we require a third and final calculus called the isotropic calculus of pseudodifferential operators. This calculus is defined by symbols $a$ which are functions on $\mathbb{R}^{2 n}$.

Definition 12. A function $a \in \mathcal{C}^{\infty}\left(\mathbb{R}^{2 n}\right)$ belongs to $S_{\text {iso }}^{m}$ if $r^{-m} a(u)$ extends smoothly to the radial compactification of $\mathbb{R}^{2 n}$, where

$$
r=|u|
$$


Or more prosaically $a$ has an asymptotic expansion

$$
a(u) \sim r^{m} \sum_{j=-\infty}^{0} a_{j}(u)
$$

where $a_{j}$ is smooth in $\mathbb{R}^{2 n} \backslash\{0\}$ and

$$
a_{j}(\lambda u)=\lambda^{j} a(u), \text { for } \lambda>0 .
$$

Exercise 12. Show that if $a \in S_{\text {iso }}^{m}$ then for any multi-index $\alpha$ there is a constant $C_{\alpha}$ so that

$$
\left|\partial_{u}^{\alpha} a(u)\right| \leq C_{\alpha}(1+|u|)^{m-|\alpha|}
$$

\section{Exercise 13.}

Show that for each multi-index $\alpha$

$$
\|a\|_{m, \alpha}=\sup \frac{\left|\partial_{u}^{\alpha} a(u)\right|}{(1+|u|)^{m-|\alpha|}}
$$

defines a semi-norm on $S_{\text {iso }}^{m}$. Show that these semi-norms define a topology on $S_{\text {iso }}^{m}$ with respect to which $S_{\text {iso }}^{m}$ is complete.

To quantize these symbols we split $\mathbb{R}^{2 n}=\mathbb{R}_{x}^{n} \times \mathbb{R}_{\xi}^{n}$ and define an operator acting on $\mathcal{S}\left(\mathbb{R}^{n}\right)$ using the Weyl formula:

$$
q_{W}(a) f=\frac{1}{[2 \pi]^{n}} \iint a\left(\frac{x+y}{2}, \xi\right) e^{i(x-y) \cdot \xi} f(y) d y d \xi .
$$

If $a$ and $b$ are Schwartz class functions then $q_{W}(a) \circ q_{W}(b)=q_{W}(c)$ where $c$ is another Schwartz class function defined by

$$
\begin{aligned}
c(u) & =a \# b(u) \\
& =\frac{1}{\pi^{2 n}} \iint a(v+u) b(w+u) e^{2 i \omega(v, w)} d v d w .
\end{aligned}
$$

with $\omega(v, w)=\tilde{v} \cdot \hat{w}-\hat{v} \cdot \tilde{w}$, the standard symplectic form on $\mathbb{R}^{2 n}$. The restrictions of the symplectic form to $\mathbb{R}^{n} \times\{0\}$ and $\{0\} \times \mathbb{R}^{n}$ are identically zero. These are complementary Lagrangian subspaces.

The operation $(a, b) \mapsto a \# b$ is determined by the symplectic structure of $\mathbb{R}^{2 n}$. It defines a non-commutative associative algebra structure on $\mathcal{S}\left(\mathbb{R}^{2 n}\right)$. It is important to note that the symbol of $q_{W}(a) \circ q_{W}(b)$ does not depend on the choice of splitting of $\mathbb{R}^{2 n}$ as a product of Lagrangian subspaces. Choosing a different such splitting defines a different quantization of $S_{\text {iso }}^{*}$ which nonetheless has the same composition formula for symbols. Using a standard integration by parts argument one can show that for any $m, m^{\prime} \in \mathbb{R}$ the \#-product extends to define a map

$$
\#: S_{\text {iso }}^{m} \times S_{\text {iso }}^{m^{\prime}} \longrightarrow S_{\text {iso }}^{m+m^{\prime}},
$$

continuous with respect to the symbolic semi- norms, such that

$$
q_{W}(a) \circ q_{W}(b)=q_{W}(a \# b) .
$$

Exercise 14. Use (24) to extend the \#-product to a map \# : $S_{\text {iso }}^{m} \times S_{\text {iso }}^{m^{\prime}} \longrightarrow S_{\text {iso }}^{m+m^{\prime}}$ continuous with to respect the symbolic semi-norms.

Exercise 15. If $a \in S_{\text {iso }}^{m}$ and $b \in S_{\text {iso }}^{m^{\prime}}$ show that $a \# b$ has an asymptotic expansion

$$
a \# b(x, \xi) \sim \sum_{k=0}^{\infty} \frac{i^{k}}{2^{k} k !}\left[D_{x} \cdot D_{\eta}-D_{y} \cdot D_{\xi}\right]^{k} a(x, \xi) b(y, \eta) \uparrow_{y=x, \eta=\xi},
$$

where $D_{x}=-i\left(\partial_{x_{1}}, \ldots, \partial_{x_{n}}\right)$. 
Definition 13. An isotropic symbol $a$ of order $m$ is elliptic if there is an isotropic symbol $b$ of order $-m$ such that

$$
a \# b-1 \in \mathcal{S}\left(\mathbb{R}^{2 n}\right) \text { and } b \# a-1 \in \mathcal{S}\left(\mathbb{R}^{2 n}\right) .
$$

An isotropic symbol $a$ of order $m$ is invertible if there is an isotropic symbol $b$ of order $-m$ so that $a \# b=b \# a=1$.

Invertibility is a stronger condition than ellipticity!

Exercise 16. If $a \in \mathcal{S}\left(\mathbb{R}^{2 n}\right)$ show that $1+a$ is an elliptic isotropic symbol though it may fail to be invertible.

The principal symbol of a Heisenberg pseudodifferential operator is a homogeneous function on the cotangent bundle. This means that it is determined by its restriction to any hypersurface transverse to the orbits of parabolic $\mathbb{R}_{+}$-action. For example the principal symbol is determined by its restriction to $\rho=1$. It turns out to be more convenient to use a different transversal hypersurface: we use instead the bundles of affine hyperplanes

$$
H^{ \pm}=\left\{\sigma_{0}= \pm 1\right\} .
$$

We refer to these as the upper and lower hyperplanes, respectively. The subspace $\sigma_{0}=0$ is invariant under the parabolic action and so orbits in this set do not meet either affine plane. However this does not lead to difficulties since these orbits lie in the closure of the orbits which do intersect $H^{ \pm}$. As the symbols are continuous functions their values on the orbits which lie in $\sigma_{0}=0$ are determined. For an operator $A \in \Psi_{\mathrm{H}}^{m}(X)$ we let

$$
\sigma_{m}^{\mathrm{H}}(A)( \pm)\left(x, \sigma^{\prime}\right)=\sigma_{m}^{\mathrm{H}}(A)\left(x, \pm 1, \sigma^{\prime}\right) .
$$

For each $x$ the affine hyperplanes, $H_{x}^{ \pm}$are linearly isomorphic to $\mathbb{R}^{2 n}$. A function $a \in$ $\mathcal{C}^{\infty}\left(H^{ \pm}\right)$, that is a smooth function on the total space of the affine hyperplane field $H^{ \pm}$, belongs to $S_{\text {iso }}^{m}\left(H^{ \pm}\right)$if, for each $x \in X, a(x, \cdot) \in S_{\text {iso }}^{m}\left(H_{x}^{ \pm}\right)$. In other words elements of $S_{\text {iso }}^{m}\left(H^{ \pm}\right)$are the smooth sections of a vector bundle with fiber $S_{\text {iso }}^{m}\left(\mathbb{R}^{2 n}\right)$.

If $\theta$ is the contact form then $\pm d \theta$ induces a symplectic structures on $H^{ \pm}$. This means that for each $x$ we can define a \#-product such that

$$
\#_{ \pm}: S_{\text {iso }}^{m}\left(H_{x}^{ \pm}\right) \times S_{\text {iso }}^{m^{\prime}}\left(H_{x}^{ \pm}\right) \longrightarrow S_{\text {iso }}^{m+m^{\prime}}\left(H_{x}^{ \pm}\right) .
$$

Note that we use $+d \theta$ to define the \#-product on $H^{+}$and $-d \theta$ to define it on $H^{-}$. This product makes $S_{\text {iso }}^{*}\left(H^{ \pm}\right)$into a bundle of algebras. The set of smooth sections of $H^{ \pm}$ which are Schwartz class functions on each fibre is denoted $\mathcal{S}\left(H^{ \pm}\right)$. These are ideals in the bundle of isotropic algebras:

$$
\mathcal{S}\left(H^{ \pm}\right) \#_{ \pm} S_{\text {iso }}^{*}\left(H^{ \pm}\right), \quad S_{\text {iso }}^{*}\left(H^{ \pm}\right) \#_{ \pm} \mathcal{S}\left(H^{ \pm}\right) \subset \mathcal{S}\left(H^{ \pm}\right) .
$$

With these preliminaries we can state the symbolic composition result for the Heisenberg calculus. Let $A \in \Psi_{\mathrm{H}}^{m}(X)$; it is a simple consequence of Heisenberg homogeneity that

$$
\sigma_{m}^{\mathrm{H}}(A)( \pm) \in S_{\text {iso }}^{2 m}\left(H_{ \pm}\right) .
$$

Theorem 6. If $A \in \Psi_{\mathrm{H}}^{m}(X)$ and $B \in \Psi_{\mathrm{H}}^{m^{\prime}}(X)$ then $A \circ B \in \Psi_{\mathrm{H}}^{m+m^{\prime}}(X)$ and

$$
\sigma_{m+m^{\prime}}^{\mathrm{H}}(A \circ B)( \pm)=\sigma_{m}^{\mathrm{H}}(A)( \pm) \#_{ \pm} \sigma_{m^{\prime}}^{\mathrm{H}}(B)( \pm) .
$$

This is a composition formula for the principal symbol; it is a coordinate invariant statement. This theorem appears in [32] and [4]. A proof of the composition theorem in the spirit of this discussion is given in [14]. In [4] and [32] mapping properties for operators in $\Psi_{\mathrm{H}}^{*} X$ are proved. A consequence of these results is the following proposition. 
Proposition 8. If $A \in \Psi_{\mathrm{H}}^{0}(X)$ then $A$ defines a bounded operator on $L^{2}(X)$. If $A \in$ $\Psi_{\mathrm{H}}^{m}(X)$ for an $m<0$ then $A$ defines a compact operator on $L^{2}(X)$.

Definition 14. An operator $A \in \Psi_{\mathrm{H}}^{m}(X)$ is elliptic if the symbols $\sigma_{m}^{\mathrm{H}}(A)( \pm)$ are invertible elements of the isotropic algebra.

It does not suffice for $\sigma_{m}^{\mathrm{H}}(A)( \pm)$ to be elliptic elements of the isotropic algebra.

If $A$ is elliptic then there exists $B_{0} \in \Psi_{\mathrm{H}}^{-m}(X)$ such that

$$
\sigma_{m}^{\mathrm{H}}(A)( \pm) \#_{ \pm} \sigma_{-m}^{\mathrm{H}}\left(B_{0}\right)( \pm)=\sigma_{-m}^{\mathrm{H}}\left(B_{0}\right)( \pm) \#_{ \pm} \sigma_{m}^{\mathrm{H}}(A)( \pm)=1 .
$$

Using the composition properties of the Heisenberg calculus this is easily shown to imply that there is an element $B \in \Psi_{\mathrm{H}}^{-m}(X)$ such that

$$
A \circ B-\mathrm{Id}, \quad B \circ A-\mathrm{Id} \in \Psi_{\mathrm{H}}^{-\infty} X .
$$

As noted above we can quantize an isotropic symbol defined on $\mathbb{R}^{2 n}$ and obtain an operator which acts on $\mathcal{S}\left(\mathbb{R}^{n}\right)$. If $q_{W}(a)$ denotes the operator defined by the symbol $a$ then the \#-product satisfies

$$
q_{W}(a) \circ q_{W}(b)=q_{W}(a \# b) .
$$

From formula (25) it is evident that this quantization requires a splitting of $\mathbb{R}^{2 n}$ as $\mathbb{R}^{n} \times$ $\mathbb{R}^{n}$. This is not an arbitrary splitting, the two factors must be complementary Lagrangian subspaces. Suppose that $a \in S_{\text {iso }}^{m}$. Quantization is a useful tool as it allows the application of operator theoretic techniques to determine if $a$ is an invertible element of the isotropic symbol algebra. In [32] it is show there exists an $b \in S_{\text {iso }}^{-m}$ such that

$$
a \# b=b \# a=1,
$$

if and only if "the operator" $q_{W}(a): \mathcal{S}\left(\mathbb{R}^{n}\right) \rightarrow \mathcal{S}\left(\mathbb{R}^{n}\right)$ is invertible. Of course this operator depends on the choice of Lagrangian splitting used to define the quantization, however, the invertibility or non-invertibility of $q_{W}(a)$ does not.

In general the hyperplane bundles $H^{ \pm}$do not admit global splittings as products of Lagrangian subbundles. For most applications this is of no real importance: If $U \subset X$ is a Darboux coordinate neighborhood then we can choose smoothly varying local Lagrangian splittings for $H^{ \pm}\left\lceil_{U}\right.$. We can then quantize symbols $\sigma_{m}^{\mathrm{H}}(A)( \pm)(x)$ for $x \in U$, obtaining smooth families of operators $q_{W}\left(\sigma_{m}^{\mathrm{H}}(A)( \pm)(x)\right)$ which act on $\mathcal{S}\left(\mathbb{R}^{n}\right)$. These operators are called the model operators for $A$. As a consequence of the result for $S_{\text {iso }}^{*}$ the symbols $\sigma_{m}^{\mathrm{H}}(A)( \pm)(x)$ are invertible with respect to the \#-product structure if and only if the model operators

$$
q_{W}\left(\sigma_{m}^{\mathrm{H}}(A)( \pm)(x)\right): \mathcal{S}\left(\mathbb{R}^{n}\right) \longrightarrow \mathcal{S}\left(\mathbb{R}^{n}\right)
$$

are invertible. While the "model operators" themselves depend on the choice of Lagragian splitting, their invertibility or non-invertibility does not. For many important operators in the Heisenberg calculus it is possible to explicitly compute the model operators and thereby determine their ellipticity or non-ellipticity.

\section{Application of the Heisenberg Calculus to SEVERAl Complex VARIABLES}

For the applications of the Heisenberg calculus to several complex variables as well as index theory there is one operator of central importance: the sum of squares:

$$
\mathcal{L}_{\alpha}=\sum_{j=1}^{n} X_{j}^{2}+Y_{j}^{2}+i \alpha T .
$$


If $\alpha$ is real then, with respect to the flat volume form $d t d x d y, \mathcal{L}_{\alpha}$ is a formally self adjoint operator. Its Heisenberg principal symbol is given by

$$
\sigma_{2}^{\mathrm{H}}\left(\mathcal{L}_{\alpha}\right)(\sigma)=-\sum_{j=1}^{2 n} \sigma_{j}^{2}-\alpha \sigma_{0} .
$$

This implies that

$$
\sigma_{2}^{\mathrm{H}}\left(\mathcal{L}_{\alpha}\right)(\sigma)( \pm)=-\sum_{j=1}^{2 n} \sigma_{j}^{2} \mp \alpha .
$$

For a moment, let $\sigma^{\prime}=(y, \eta)$ denote variables splitting $H_{ \pm}$into complementary Lagrangian subspaces. The symbol of $\mathcal{L}_{\alpha}$ becomes:

$$
\sigma_{2}^{\mathrm{H}}\left(\mathcal{L}_{\alpha}\right)( \pm)=-\left(|\eta|^{2}+|y|^{2}\right) \mp \alpha .
$$

Quantizing this symbol as described we find that

$$
q_{W}\left(\sigma_{2}^{\mathrm{H}}\left(\mathcal{L}_{\alpha}\right)( \pm)\right)=\Delta-|y|^{2} \mp \alpha .
$$

The operator $\Delta-|y|^{2}$ is of course the quantum harmonic oscillator. Before analyzing this operator we explain the connection between $\mathcal{L}_{\alpha}$ and the CR-structure on the Heisenberg group.

On $\mathbb{H}^{n}$, there is a natural CR-structure with

$$
T^{0,1} \mathbb{H}^{n}=\operatorname{span}\left\{\bar{Z}_{j}=\frac{1}{2}\left(X_{j}+i Y_{j}\right)=\partial_{z_{j}}-\frac{i}{2} \bar{z}_{j} \partial_{t}\right\} .
$$

With respect to this CR-structure

$$
\bar{\partial}_{b} f=\sum \bar{Z}_{j} f d \bar{z}_{j}
$$

If $\omega=\sum g_{j} d z_{j}$, then $\bar{\partial}_{b}^{*}(\omega)=-\sum Z_{j} g_{j}$. So the $\square_{b}$-operator is given by

$$
\begin{aligned}
\square_{b} f=\bar{\partial}_{b}^{*} \bar{\partial}_{b} f & =\sum Z_{j} \bar{Z}_{j} f \\
& =-\frac{1}{4}\left(\sum_{j=1}^{n} X_{j}^{2}+Y_{j}^{2}+i\left(\left[X_{j}, Y_{j}\right]\right)\right. \\
& =-\frac{1}{4}\left(\sum_{j=1}^{n} X_{j}^{2}+Y_{j}^{2}-i n T\right) \\
& =-\frac{1}{4} \mathcal{L}_{-n} .
\end{aligned}
$$

This is the flat computation. On any strictly pseudoconvex CR-manifold $X$ we similarly define the $\square_{b}$-operator on functions by

$$
\square_{b}=\bar{\partial}_{b}^{*} \bar{\partial}_{b} .
$$

About any given point $x$ one can choose local coordinates so that, in these coordinates the $\square_{b}$-operator on functions is given by

$$
\square_{b}=-\frac{1}{4} \mathcal{L}_{-n}+\text { l. o.t. . }
$$

Here l.o.t. are terms of order less than 2 as well as terms of order 2 with coefficients vanishing at $x$. Thus

$$
\sigma_{2}^{\mathrm{H}}\left(\square_{b}\right)( \pm)(x)=-\frac{1}{4} \sigma_{2}^{\mathrm{H}}\left(\mathcal{L}_{-n}\right)( \pm)(x) .
$$

This explains why the analysis of the sum of squares operator is so important in several complex variables. 
In addition to $\bar{\partial}_{b}$ on functions there is a complex, analogous to the $\bar{\partial}$-complex which is defined on a CR-manifold, $X$. For each $0 \leq q \leq n$ we define a vector bundle $\Lambda_{b}^{0, q} X$ with fiber $\Lambda^{q}\left(T_{x}^{0,1} X\right)^{\prime}$. Invariantly this is a quotient of the complexified bundle $\Lambda^{q} T^{*} X \otimes \mathbb{C}$. If $\theta$ denotes a contact form with $T$ the corresponding "Reeb" vector field then $\left(T^{0,1} X\right)^{\prime}$ can be identified with the annihilator of $T^{1,0} X \oplus \mathbb{C} T$. If $P_{1}$ denotes the projection into this annihilator then for $f \in \mathcal{C}^{\infty}(X)$ we have a representation of the $\bar{\partial}_{b}$-operator:

$$
\bar{\partial}_{b} f=P_{1}(d f) .
$$

Similarly for each $q$ we get a projection, $P_{q}$ into a subbundle of $\Lambda^{q} T^{*} X \otimes \mathbb{C}$ which represents $\Lambda_{b}^{0, q} X$. If $\omega$ is a section of $\Lambda_{b}^{0, q-1} X$ then

$$
\bar{\partial}_{b} \omega=P_{q}(d \omega) .
$$

It is a consequence of the integrability of the CR-structure that $\bar{\partial}_{b} \circ \bar{\partial}_{b}=0$. The complex,

$$
\mathcal{C}^{\infty}\left(X ; \Lambda_{b}^{0,0}\right) \stackrel{\bar{\partial}_{b}}{\longrightarrow} \mathcal{C}^{\infty}\left(X ; \Lambda_{b}^{0,1}\right) \stackrel{\bar{\partial}_{b}}{\longrightarrow} \cdots \stackrel{\bar{\partial}_{b}}{\longrightarrow} \mathcal{C}^{\infty}\left(X ; \Lambda_{b}^{0, n}\right)
$$

is called the Kohn-Rossi or $\bar{\partial}_{b}$-complex.

Exercise 17. Show that the integrability of a CR-structure is equilvalent to $\bar{\partial}_{b}^{2}=0$.

In general if $\alpha \in \mathcal{C}^{\infty}\left(M, \Lambda_{b}^{0, q}\right)$, then we get the following expression for $\bar{\partial}_{b} \alpha$ :

$$
\bar{\partial}_{b} \alpha=\sum \bar{Z}_{j} f_{\beta} \bar{\omega}_{j} \wedge \bar{\omega}^{\beta}+\sum_{\beta} f_{\beta} P_{q+1}\left(d \bar{\omega}^{\beta}\right) .
$$

Choosing an hermitian metric on $\Lambda_{b}^{0,1}$ we can define the formal adjoint of $\bar{\partial}_{b}$ which is denoted

$$
\bar{\partial}_{b}^{*}: \mathcal{C}^{\infty}\left(X ; \Lambda_{b}^{0, q+1}\right) \longrightarrow \mathcal{C}^{\infty}\left(X ; \Lambda_{b}^{0, q}\right) .
$$

The $\square_{b}$-operator on $(0, q)$-forms is defined by

$$
\square_{b} \alpha=\left(\bar{\partial}_{b}^{*} \bar{\partial}_{b}+\bar{\partial}_{b} \bar{\partial}_{b}^{*}\right) \alpha .
$$

A calculation shows that

$$
\begin{gathered}
\square_{b} \alpha=-\sum_{\beta}\left\{\sum_{j=1}^{n}\left(Z_{j} \bar{Z}_{j}+\bar{Z}_{j} Z_{j}\right)+\sum_{j \notin \beta}\left[Z_{j}, \bar{Z}_{j}\right]-\sum_{j \in \beta}\left[Z_{j}, \bar{Z}_{j}\right]\right\} f_{\beta} \bar{\omega}^{\beta} \\
\quad+\text { lower order terms, }
\end{gathered}
$$

see [4]. Using the coordinates introduced above, we obtain that at the center $x$ of this coordinate system,

$$
\begin{aligned}
\square_{b} \alpha=-\frac{1}{4} \sum_{\beta}( & \left.\sum X_{j}^{2}+Y_{j}^{2}+-i(n-2 q) T\right) f_{\beta} \bar{\omega}^{\beta} \\
& + \text { l. o.t. }
\end{aligned}
$$

This implies that at $x$ :

$$
\sigma_{2}^{\mathrm{H}}\left(\square_{b}^{0, q}\right)( \pm)(x)=-\frac{1}{4} \sigma_{2}^{\mathrm{H}}\left(\mathcal{L}_{-(n-2 q)} \otimes I d_{\Lambda_{b}^{0, q}}\right)( \pm)(x) .
$$

Once again we see that to use the Heisenberg calculus to analyze $\square_{b}^{0, q}$, the first step is to analyze the sum of squares operator, $\mathcal{L}_{2 q-n}$. We now analyze the quantum harmonic oscillator, as it is the model operator for $\sigma_{2}^{\mathrm{H}}\left(\mathcal{L}_{0}\right)$. 


\section{THE QUANTUM HARMONIC OSCILLATOR AND $\square_{b}$}

By using separation of variables, the theory of $n$-dimensional harmonic oscillator can be reduced to the one dimensional case,

$$
\mathbf{H}=-\partial_{y}^{2}+y^{2} .
$$

To do this case we introduce the annihilation and creation operators:

$$
C=y+\partial_{y}, \quad C^{*}=y-\partial_{y}
$$

Elementary computations show that

$$
\begin{aligned}
& C^{*} C+\mathrm{Id}=C C^{*}-\mathrm{Id}=\mathbf{H}, \\
& {[C, \mathbf{H}]=2 C, \quad\left[C^{*}, \mathbf{H}\right]=-2 C^{*} .}
\end{aligned}
$$

If $\mathbf{H} f=\lambda f$ then using (29) we deduce that

$$
\begin{aligned}
\mathbf{H} C^{*} f & =(\lambda+2) C^{*} f, \\
\mathbf{H} C f & =(\lambda-2) C f .
\end{aligned}
$$

We see that $C^{*}$ increases the energy level, it is called the creation operator. Its formal adjoint, $C$ is called the annihilation operator.

It is a standard result that $\mathbf{H}$ acting on $\mathcal{C}_{\mathrm{c}}^{\infty}(\mathbb{R})$ is an essentially self adjoint operator. Its closure is a self adjoint operator with pure point spectrum, see [33]. The $L^{2}$-function

$$
f_{0}=\exp \left(-\frac{y^{2}}{2}\right)
$$

satisfies

$$
\mathbf{H} f_{0}=f_{0} \text {. }
$$

Is it not difficult to show that

$$
\left\langle\mathbf{H}\left(\psi f_{0}\right), \psi f_{0}\right\rangle<\left\langle\psi f_{0}, \psi f_{0}\right\rangle \text { for } \psi \in \mathcal{C}_{\mathrm{c}}^{\infty}(\mathbb{R}) .
$$

This implies that the lowest eigenvalue of $\mathbf{H}$ is 1. If $g$ is a solution of $\mathbf{H} g=g$ linearly independent of $f_{0}$ then an elementary calculation shows that $|g|(y) \sim e^{y^{2} / 2}$ and therefore the lowest eigenstate is one dimensional, spanned by $f_{0}$. It is called the ground or vacuum state. We obtain the higher eigenstates by setting

$$
f_{j}(y)=\left(C^{*}\right)^{j} f_{0}
$$

Applying (29) recursively we conclude that

$$
\mathbf{H} f_{j}=(1+2 j) f_{j} .
$$

Using the commutation relations and the fact that the ground state is one-dimensional one can show that $\left\{f_{j}, j=0, \ldots, \infty\right\}$ defines orthogonal basis for $L^{2}(\mathbb{R})$.

Exercise 18. Compute the $L^{2}$-norms of the $f_{j}$,

$$
\left\langle f_{j}, f_{j}\right\rangle=\left\langle C^{j}\left(C^{*}\right)^{j} f_{0}, f_{0}\right\rangle .
$$

In the $n$-dimensional case, we have

$$
\mathbf{H}_{n}=-\Delta_{n}+|y|^{2} \text {, and } f_{0}=e^{-\frac{1}{2} \sum y_{j}^{2}} .
$$

Analogously to the one dimensional case, we have annihilation and creation operators:

$$
C_{j}=\frac{i}{2}\left(y_{j}+\partial_{y_{j}}\right), \quad C_{j}^{*}=-\frac{i}{2}\left(y_{j}-\partial_{y_{j}}\right)
$$


These operators are related to the harmonic oscillator by

$$
\sum C_{j}^{*} C_{j}=-\frac{1}{4} \sum\left(y_{j}+\partial_{y_{j}}\right)\left(y_{j}-\partial_{y_{j}}\right)=\frac{1}{4}\left(\mathbf{H}_{n}-n\right) .
$$

We also have the commutation relations:

$$
\left[C_{j}, C_{k}^{*}\right]=2 \delta_{j k}, \quad\left[C_{j}, \mathbf{H}_{n}\right]=2 C_{j}, \quad\left[C_{j}^{*}, \mathbf{H}_{n}\right]=-2 C_{j}^{*} .
$$

The higher eigenfunctions are obtained by applying the creation operators to the ground state. They are indexed by multi-indices $\mu=\left(\mu_{1}, \ldots, \mu_{n}\right)$ with $\mu_{i} \geq 0$. For such a multiindex we set

$$
f_{\mu}=\left(C^{*}\right)^{\mu} f_{0}=\left(C_{1}^{*}\right)^{\mu_{1}} \cdots\left(C_{n}^{*}\right)^{\mu_{n}} f_{0} .
$$

Using the commutation relations one easily shows that

$$
\begin{aligned}
\mathbf{H}_{n} f_{0} & =n f_{0}, \\
\mathbf{H}_{n} f_{\mu} & =(n+2|\mu|) f_{\mu} \text { where }|\mu|=\sum \mu_{i} .
\end{aligned}
$$

The spectrum of $\mathbf{H}_{n}$ is $\{n+2 k: k \in \mathbb{N} \cup\{0\}\}$.

We can now analyze the sum of squares operators

Theorem 7. The operator $\mathcal{L}_{\alpha}$ is an Heisenberg-elliptic operator provided

$$
\alpha \notin\left\{ \pm(n+2 j) \mid j \in \mathbb{N}_{0}\right\}
$$

Proof. As noted above

$$
q_{W}\left(\sigma_{2}^{\mathrm{H}}\left(\mathcal{L}_{\alpha}\right)( \pm)\right)=\frac{1}{4}\left(\mathbf{H}_{n} \mp \alpha\right)
$$

To prove the theorem it suffices to show that $\left(\mathbf{H}_{n} \mp \alpha\right)$ are invertible operators. Since this operator is self adjoint it is sufficient to show that the $L^{2}$-null space is empty. In order for

$$
\left(\mathbf{H}_{n} \mp \alpha\right) u=0
$$

to have an $L^{2}$-solution it is necessary and sufficient that $\alpha= \pm(n+2 j)$ for some $j \in \mathbb{N}_{0}$. This completes the proof of the theorem.

The symbol of $\square_{b}^{0, q}$ is diagonal with $\sigma_{2}^{\mathrm{H}}\left(\mathcal{L}_{2 q-n}\right)$ along the diagonal. Thus it is an elliptic operator in the Heisenberg calculus if and only if $q \neq 0$ or $n$. In the $q=0$ case $\square_{b}^{0,0}$ is not elliptic. This was expected, because $\operatorname{ker} \square_{b}^{0,0}=\operatorname{ker} \bar{\partial}_{b}$ is infinite dimensional for an embeddable strictly pseudoconvex CR-manifold.

The model operator for $\square_{b}^{0,0}$ on the upper hyperplane is $\frac{1}{4}\left(\mathbf{H}_{n}-n\right)$ which has a onedimensional null-space. The model operator on the lower hyperplane is $\frac{1}{4}\left(\mathbf{H}_{n}+n\right)$ which is invertible. Let $\pi_{0}$ be the operator on $L^{2}\left(\mathbb{R}^{n}\right)$ which is orthogonal projection onto the span of $f_{0}$.

$$
\pi_{0}(\psi)=f_{0} \frac{\left\langle\psi, f_{0}\right\rangle}{\left\|f_{0}\right\|^{2}}
$$

The Schwartz kernel of $\pi_{0}$ is $\left\|f_{0}\right\|^{-2}\left(f_{0}(x) \otimes f_{0}(y)\right)$. A computation using the quantization formula shows that

$$
\pi_{0}=q_{W}\left(s_{0}\right)
$$

where

$$
s_{0}=2^{n} \exp \left[-\left(|y|^{2}+|\eta|^{2}\right)\right]
$$

If $\square_{b}^{0,0}$ has a closed range in $L^{2}$ ( which is always true if $\operatorname{dim} \geq 5$ ), then using the Heisenberg calculus one can show there are operators $Q \in \Psi_{\mathrm{H}}^{-2}$ and $S \in \Psi_{\mathrm{H}}^{0}$ such that

$$
\begin{aligned}
Q \square_{b} & =\square_{b} Q=I-S, \\
S \square_{b}=\square_{b} S & =0,
\end{aligned}
$$


see [4]. The operator $Q$ is the partial inverse to $\square_{b}^{0,0}$ and $S$ is the orthogonal projection onto the null-space. $S$ is called the Szegó projector.

In Darboux coordinates the symbol of the $S$ is given by

$$
\sigma_{0}^{\mathrm{H}}(S)( \pm)=\left\{\begin{array}{l}
2^{n} \exp \left(-\left|\sigma^{\prime}\right|^{2}\right) \quad(+) \\
0 \quad(-)
\end{array}\right.
$$

As expected the $(+)$-symbol is exactly the symbol of the projection onto the ground state of a harmonic oscillator. It is an element of Heisenberg calculus of order zero. We could at this point generalize the notion of Toeplitz operator to a strictly pseudoconvex manifold. We first analyze a bit further the structures underlying the definition of the Szegó projector.

\section{FIELDS OF HARMONIC OSCILLATORS}

At the heart of the Szegó projector is a field of harmonic oscillators defined on a contact manifold. For the remainder of this section we let $X$ denote a contact manifold with contact field, $\mathcal{H}$. Let $\theta$ denote a choice of contact form and $\mathcal{H}^{\prime}$ the annihilator of the Reeb vector field. In addition $J$ denotes an almost complex structure on fibers of $\mathcal{H}^{\prime}$. The two form $d \theta$ defines a symplectic structure on the fibers of $\mathcal{H}$. Let $\omega$ denote the dual symplectic form induced on $\mathcal{H}^{\prime}$. The almost complex structure $J$ is tamed by the symplectic structure if

$$
\omega(J X, X)>0 .
$$

It is adapted to the symplectic structure if it is tamed and

$$
\omega(J X, J Y)=\omega(X, Y)
$$

for all $X, Y \in \mathcal{H}_{p}^{\prime}, \quad \forall p \in X$.

Exercise 19. Show that if the almost complex structure $J$ is defined by an integrable, strictly pseudoconvex CR-structure with underlying contact field $\mathcal{H}$ then it is adapted.

The point of making this definition is that there are adapted almost complex structures defined on any orientable contact manifold. If $g$ is a Riemannian metric on $T^{*} X$ then there is a unique endomorphism $\widetilde{\mathcal{J}}$ of $\mathcal{H}^{\prime}$ such that

$$
g(X, Y)=\omega(\widetilde{\mathcal{J}} X, Y) .
$$

From the symmetry of $g$ and skew symmetry of $\omega$ it follows that $\widetilde{\mathcal{J}}$ is a skew symmetric, real transformation with respect to $g$. This implies that the spectrum of $\widetilde{\mathcal{J}}$ is purely imaginary and eigenvalues come in conjugate pairs. Of course to diagonalize $\widetilde{\mathcal{J}}$ we need to complexify $\mathcal{H}^{\prime}$. Let $\Lambda_{b}^{0,1} \subset \mathcal{H}^{\prime} \otimes \mathbb{C}$ denote the bundle of positive eigenspaces of $\tilde{\mathcal{J}}$ and $\Lambda_{b}^{1,0}$ the negative eigenspaces. The real endomorphism $J$ underlying the splitting, $\mathcal{H}^{\prime} \otimes \mathbb{C}=\Lambda_{b}^{1,0} \oplus \Lambda_{b}^{0,1}$ is easily seen to be adapted.

Let $(x, \sigma)$ denote the coordinates on the cotangent bundle defined by a choice of Darboux coordinates.

Definition 15. With $J$ an adapted almost complex structure we define a quadratic function on $T^{*} X$ :

$$
h_{J, \omega}\left(\sigma_{0}, \sigma^{\prime}\right)=\omega\left(J \sigma^{\prime}, \sigma^{\prime}\right)
$$

This function is Heisenberg homogeneous of degree 2 and is in fact a globally defined symbol. We call this a field of harmonic oscillators. 
It is clear that the quantization of the symbol $h_{J, \omega}$ restricted to the affine planes $\sigma_{0}= \pm 1$ gives, in appropriate coordinates the harmonic oscillator on $\mathbb{R}^{n}$.

Locally we can choose a symplecto-normal basis for $T X$ : We begin with $T$, the Reeb vector field fixed by the choice of $\theta$. We then choose $X_{1}, \ldots, X_{n}$, and $Y_{1}, \ldots, Y_{n}$ such that

$$
J X_{j}=Y_{j}, J Y_{j}=-X_{j}, \text { where } j=1, \ldots, n
$$

and $\left\{X_{1}, \ldots, X_{n}, Y_{1}, \ldots, Y_{n}\right\}$ is an orthonormal basis with respect to

$$
g_{J, \theta}(V, W)=d \theta(J V, W) .
$$

Locally the field of harmonic oscillators is the principal symbol of the sum of squares operator, $-\sum_{j=1}^{n}\left[X_{j}^{2}+Y_{j}^{2}\right]$. In general this operator is is not globally defined even though its principal symbol is.

Exercise 20. Suppose that $J_{1}$ and $J_{2}$ are adapted almost complex structures. Show that there exists a symplecto-normal basis as above such that if $(y, \eta)$ are the dual coordinates defined in $T^{*} X$ then

$$
\begin{aligned}
& h_{J_{1}, \omega}(y, \eta)=\sum_{j=1}^{n} y_{j}^{2}+\eta_{j}^{2}, \\
& h_{J_{2}, \omega}(y, \eta)=\sum_{j=1}^{n} \mu_{j} y_{j}^{2}+\mu_{j}^{-1} \eta_{j}^{2} .
\end{aligned}
$$

Here $\left\{\mu_{1}, \ldots, \mu_{n}\right\}$ are positive numbers.

The symbol of the projection onto the ground state of the field of harmonic oscillators is

$$
s_{0}=e^{-\frac{h_{\omega, J}}{\sigma_{0}}} .
$$

Let $s_{k}$ be the symbol of the projection onto the $k^{\text {th }}$ eigenspace of the field of harmonic oscillators. The symbols of generalized Szegô projectors vanish on the lower hyperplane. In the sequel we use $\sigma_{0}^{\mathrm{H}}(S)$ to denote $\sigma_{0}^{\mathrm{H}}(+)(S)$ with the understanding that $\sigma_{0}^{\mathrm{H}}(-)(S) \equiv$ 0 . Indeed the full symbol of $S$ vanishes in the lower half space.

Definition 16. A generalized Szegó projector is any operator $S \in \Psi_{\mathrm{H}}^{0}$ such that

(a) $S^{2}=S$,

(b) $\sigma_{0}^{\mathrm{H}}(S)=s_{0}$ for some choice of adapted almost complex structure $J$.

Definition 17. A generalized Szegó projector at level $k$ is any operator $S \in \Psi_{\mathrm{H}}^{0}$ such that

$$
S^{2}=S, \text { and } \sigma_{0}^{\mathrm{H}}(S)=s_{k}
$$

for some choice of adapted almost complex structure $J$.

Definition 18. A generalized Szegö projector to level $N$ is any projection $S \in \Psi_{\mathrm{H}}^{0}$ such that

$$
\sigma_{0}^{\mathrm{H}}(S)=\sum_{j=0}^{N} s_{j} .
$$

Remark 1. The notion of a generalized Szegó projector appears in the work of Boutet de Monvel and Guillemin on the quantization of contact structures, see [8].

Proposition 9. Suppose that $S, S^{\prime}$ are two generalized Szegó projections at the level 0. Then the map

$$
S: \text { range } S^{\prime} \rightarrow \text { range } S
$$

is a Fredholm operator. 
Remark 2. These projections can be quite different and do not, in general have the same principal symbols.

Proof. The range of each operator is closed, hence it is itself a Hilbert space. All we have to do is to find an inverse up to compact error. Denote the respective symbols by

$$
\sigma(S)=s_{0}, \text { and } \sigma\left(S^{\prime}\right)=s_{0}^{\prime} .
$$

In virtue of (34) there are Darboux coordinates $(x, \eta)$ such that when we quantize these symbols to obtain operators on $L^{2}\left(\mathbb{R}^{n}\right)$ they have Schwartz kernels of the form

$$
\pi=V(x) \otimes V(y), \quad \pi^{\prime}=V^{\prime}(x) \otimes V^{\prime}(y) .
$$

where $V$ and $V^{\prime}$ are pointwise positive functions. The symbol of $S S^{\prime}$ is

$$
\sigma_{0}^{\mathrm{H}}\left(S S^{\prime}\right)=\left\langle V, V^{\prime}\right\rangle V(x) \otimes V^{\prime}(y) .
$$

Because $\left\langle V, V^{\prime}\right\rangle$ is the $L^{2}$ inner product of strictly positive functions, it is non-vanishing. We can now define an approximate inverse. With $u=\left\langle V, V^{\prime}\right\rangle^{2}$ we define

$$
F:=S^{\prime} \frac{1}{u} \in \Psi_{\mathrm{H}}^{0}
$$

The principal symbol of the composition is

$$
\sigma_{0}^{\mathrm{H}}\left(F S S^{\prime}\right)=\sigma_{0}^{\mathrm{H}}\left(S^{\prime}\right) .
$$

As $S^{\prime} \uparrow_{\text {range } S^{\prime}}=$ Id we see that the composition

$$
F S S^{\prime}: \text { range } S^{\prime} \rightarrow \operatorname{range} S^{\prime}
$$

is the identity up to a compact error. Using a similar construction leads to an approximate right inverse. This proves the proposition.

Definition 19. The relative index is defined to be the Fredholm index of the restriction

$$
\operatorname{R}-\operatorname{Ind}\left(S, S^{\prime}\right)=\operatorname{Ind}\left(S^{\prime} S: \text { range } S \rightarrow \operatorname{range} S^{\prime}\right) .
$$

The relative index for generalized Szegó projectors satisfies a co-cycle relation:

Proposition 10. If $S, S^{\prime}, S^{\prime \prime}$ are generalized Szegó projectors at level zero then then

$$
\mathrm{R}-\operatorname{Ind}\left(S, S^{\prime \prime}\right)=\mathrm{R}-\operatorname{Ind}\left(S, S^{\prime}\right)+\mathrm{R}-\operatorname{Ind}\left(S^{\prime}, S^{\prime \prime}\right) \text {. }
$$

Remark 3. Relative indices are a basic tool for relating one kind of index problem to another. The relative index labels the path components of the space of generalized Szegó projections. That is two generalized Szegó projectors have relative index zero if and only if there is a smooth path through generalized Szegó projectors from one to the other. This result is proved in [13]. The notion of relative indices appears in the literature on index formulæ for boundary value problems. In the context of boundary value problems, the projections are classical pseudodifferential operators often assumed to have the same principal symbol. The relative index was introduced in the context of the Szegô projectors defined by integrable CR-structures in [11] and in the generality considered here in [13].

We now define generalized Toeplitz operators.

Definition 20. A generalized Toeplitz operator is an operator of the form

$$
T_{A} f=S A S f
$$

where $S$ is a generalized Szegő projector and $A \in \Psi_{\mathrm{H}}^{m}(M)$. 
Such operators were considered in [8] but $A$ is assumed to be a classical pseudodifferential. As we shall see this is, up to lower order terms the same class of operators. As before for generalized Szegó projectors, we can define generalized Toeplitz operators at the level $k$ and to the level $N$.

Definition 21. A generalized Toeplitz operator at level $k$ is a operator of the form

$$
T_{A} f=S A S f
$$

where $S$ is a generalized Szegó projector at level $k$ and $A \in \Psi_{\mathrm{H}}^{m}(M)$.

Definition 22. A generalized Toeplitz operator to level $N$ is a operator of the form

$$
T_{A} f=S A S f
$$

where $S$ is a generalized Szegő projector to level $N$ and $A \in \Psi_{\mathrm{H}}^{m}(M)$.

In the sequel the unmodified term "generalized Toeplitz operator" refers to a generalized Toeplitz operator at level 0 . In our formulation it is very easy to see that Toeplitz operators form an algebra:

$$
T_{A} T_{B}=S A S S B S=S(A S B) S .
$$

Since $A S B \in \Psi_{\mathrm{H}}^{*}(X)$ if $A, B \in \Psi_{\mathrm{H}}^{*}(X)$ the claim follows immediately.

Proposition 11. Let $S$ be the generalized Szegó projection (at the level 0) and let $A$ be an element of $\Psi_{\mathrm{H}}^{0}$, then there exists a smooth function $a$ such that

$$
S A S-S M_{a} S \in \Psi_{\mathrm{H}}^{-1}(M) \text {. }
$$

Here $M_{a}$ is the multiplication operator $M_{a} f=a f$.

The function $a$ is given by

$$
a=\int s_{0} \# \sigma_{0}^{\mathrm{H}}(A)(+) \# s_{0} \omega^{n} .
$$

The symbol $s_{0}$ is defined in (35). If $A$ is a classical pseudodifferential operator then $a$ is just the principal symbol of $A$ restricted to the positive contact direction. If $a$ is a smooth function then we denote the operator $S M_{a} S$ by $T_{a}$.

For $a$, a smooth function, and $B \in \Psi_{\mathrm{H}}^{m}(X)$ a straightforward computation shows that

$$
\sigma_{m}^{\mathrm{H}}\left(M_{a} B\right)=\sigma_{m}^{\mathrm{H}}\left(B M_{a}\right)=a \sigma_{m}^{\mathrm{H}}(B) .
$$

This in turn implies that $\sigma_{m}^{\mathrm{H}}\left(\left[M_{a}, B\right]\right)=0$ and therefore

$$
\left[M_{a}, B\right] \in \Psi_{\mathrm{H}}^{m-1}(X) .
$$

Applying this to Toeplitz operators we see that

$$
\begin{aligned}
T_{a} T_{b} & =S M_{a} S M_{b} S \\
& =S M_{a b} S+S\left[M_{a}, S\right] S M_{b} S .
\end{aligned}
$$

As $\left[M_{a}, S\right] \in \Psi_{\mathrm{H}}^{-1}$ it follows that

$$
T_{a} T_{b}-T_{a b} \in \Psi_{\mathrm{H}}^{-1}
$$

A Toeplitz operator, $T_{A}=S A S$ of any type is Fredholm if it is a Fredholm operator from range $S$ to itself. The index of such an operator is always understood as the index of this restriction. An immediate consequence of this computation is

Proposition 12. If $a \in \mathcal{C}^{\infty}(X)$ is non vanishing, then the generalized Toeplitz operator $T_{a}$ is a Fredholm operator. 
Proof. Proposition 8 implies that operators in $\Psi_{\mathrm{H}}^{-1}(X)$ are compact. It therefore follows from (37) that $T_{a^{-1}}$ is an approximate inverse for $T_{a}: L^{2}(X) \rightarrow L^{2}(X)$.

If $a \in \mathcal{C}^{\infty}(X)$ is non-vanishing then it is easy to see that for any generalized Szegő projector, $S$ the operator $a^{-1} S a$ is another generalized Szegó projector with the same principal symbol. We can relate the index of $T_{a}$ to a relative index:

Proposition 13. If $S$ is a generalized Szegő projector at level 0 and $a \in \mathcal{C}^{\infty}(X)$ is nonvanishing then

$$
\operatorname{Ind}\left(T_{a}\right)=\mathrm{R}-\operatorname{Ind}\left(S, a^{-1} S a\right) .
$$

Exercise 21. Use this proposition and the co-cycle relation to prove that that $\operatorname{Ind}\left(T_{a}\right)$ does not depend on the choice of $S$.

Proofs of these results can be found in [13].

\section{Vector BUNDLE COEFFICIENTS AND THE ATIYAH-SINGER INDEX THEOREM}

So far we have discussed operators acting on functions. We can easily extend this formalism to define pseudodifferential operators acting on sections of vector bundles. This is very important for applications to index theory. We first review the classical case. Let $E \rightarrow X$ and $F \rightarrow X$ be complex vector bundles with fiber dimensions $k$ and $l$ respectively. Let $U$ be a coordinate neighborhood on $X$ over which $E$ and $F$ are trivialized. In $U$ we have bundle maps identifying

$$
E \uparrow_{U} \simeq U \times \mathbb{C}^{k}, \quad F \uparrow_{U} \simeq U \times \mathbb{C}^{l} .
$$

If $P$ is a linear differential operator carrying sections of $E$ to sections of $F$ then, in terms of these trivializations, $P \uparrow_{U}$ is represented by a $l \times k$ matrix of differential operators $\left[P_{i j}\left(x, D_{x}\right)\right]$. By analogy with the scalar case we define the symbol of $P$ by formally replacing $D_{x}$ by the fiber coordinate in the cotangent bundle: $\sigma(P)(x, \xi)=\left[P_{i j}(x, \xi)\right]$. If $\left(s_{1}, \ldots, s_{k}\right)$ are the coordinates of $s \in \mathcal{C}_{\mathrm{c}}^{\infty}(U ; E)$ with respect to the given trivialization then we can represent the action of $P$ on $s$ using the Fourier transform in the local coordinates by

$$
(P s)_{i}(x)=\frac{1}{[2 \pi]^{n}} \int_{\mathbb{R}^{n}} e^{i \xi \cdot x} \sum_{j=1}^{k}\left[P_{i j}(x, \xi)\right] \hat{s}_{j}(\xi) d \xi, \quad i=1, \ldots, l .
$$

From this formula it is apparent that for each $(x, \xi)$ the matrix $\left[P_{i j}(x, \xi)\right]$ is an element of $\operatorname{hom}\left(E_{x}, F_{x}\right)$. In other words $\sigma(P)(x, \xi)$ is a section of the vector bundle

$$
\operatorname{hom}\left(\pi^{*}(E), \pi^{*}(F)\right) \longrightarrow T^{*} X .
$$

Here $\pi: T^{*} X \rightarrow X$ is the canonical projection.

With this as motivation we define pseudodifferential operators of order $m$ carrying sections of $E$ to sections of $F$ as operators $A: \mathcal{C}^{\infty}(X ; E) \rightarrow \mathcal{C}^{\infty}(X ; F)$ such that if $\varphi \in \mathcal{C}_{\mathrm{c}}^{\infty}(U)$ then, in terms of local trivializations of $E \uparrow_{U}$ and $F \uparrow_{U}$ we have the representation

$$
\varphi A \varphi s=\frac{1}{[2 \pi]^{n}} \int_{\mathbb{R}^{n}} e^{i \xi \cdot x} a(x, \xi) \widehat{\varphi s}(\xi) d \xi .
$$

In terms of local trivializations of $E$ and $F, a$ is represented by an $l \times k$ matrix of symbols $a_{i j} \in S_{\mathrm{KN}}^{m}(U)$. If $\varphi, \psi \in \mathcal{C}_{\mathrm{c}}^{\infty}(X)$ have disjoint support that $\varphi A \psi$ is a smoothing operator. We denote this class of operators by $\Psi_{\mathrm{KN}}^{m}(X ; E, F)$. More invariantly $a$ is a symbol of order $m$ taking values in $\operatorname{hom}\left(\pi^{*}(E), \pi^{*}(F)\right)$. As with scalar operators the full symbol transforms in a very complicated way under changes of coordinate, however the 
leading term in the asymptotic expansion of the symbol is just a homogeneous section of $\operatorname{hom}\left(\pi^{*}(E), \pi^{*}(F)\right)$ defined in the complement of the zero section of $T^{*} X$. This is the principal symbol of $A$ which we continue to denote by $\sigma_{m}(A)$.

Using the same local discussion we define Heisenberg operators carrying sections of $E$ to sections of $F$. The single modification is that, in terms of local trivializations, the section $a$ is represented by a matrix of symbols $a_{i j} \in S_{\mathrm{H}}^{m}(U)$. The principal symbol is again defined as the leading term in the asymptotic expansion of $a$ in Heisenberg homogeneous terms. Invariantly it takes values in $\operatorname{hom}\left(\pi^{*}(E), \pi^{*}(F)\right)$. We denote the Heisenberg operators of order $m$ acting between sections of $E$ and $F$ by $\Psi_{\mathrm{H}}^{m}(X ; E, F)$. If $E=F$ we abbreviate this notation to $\Psi_{\mathrm{H}}^{m}(X ; E)$.

Let $E \rightarrow X$ be a complex vector bundle over a contact manifold. A generalized Szegó projection, at the level 0 , acting on the sections of $E$ is any projection operator $S_{E} \in$ $\Psi_{\mathrm{H}}^{0}(X ; E)$ such that

$$
\sigma_{0}^{\mathrm{H}}\left(S_{E}\right)=s_{0} \otimes \operatorname{Id}_{E} .
$$

Here $s_{0}$ is the symbol of a scalar generalized Szegó projector. Similarly, we define generalized Szegó projections at level $k$ and to level $N$ acting on sections of $E$ as projection operators $S_{E} \in \Psi_{\mathrm{H}}^{0}(X ; E)$ with the following properties

$$
\begin{aligned}
& S_{E} \text { is a projection at the level } k \text { if } \sigma_{k}^{\mathrm{H}}\left(S_{E}\right)=s_{k} \otimes \operatorname{Id}_{E} \\
& S_{E} \text { is a projection to the level } N \text { if } \sigma_{N}^{\mathrm{H}}\left(S_{E}\right)=\left(\sum_{1}^{N} s_{k}\right) \otimes \operatorname{Id}_{E} .
\end{aligned}
$$

Here $s_{k}$ denotes the symbol of a scalar, generalized Szegó projector at level $k$. Once again for each family of generalized Szegő projectors acting on sections of $E$ there is a corresponding family of Toeplitz operators $T_{A}=S_{E} A S_{E}$ where $A \in \Psi_{\mathrm{H}}^{0}(M ; E)$.

Before considering index formulæ for Heisenberg operators we first review the AtiyahSinger theorem for classical, elliptic pseudodifferential operators. As above we let $Y$ be a compact manifold of dimension $n$ and $E, F$ be vector bundles over $Y$. Let $P \in$ $\Psi^{0}(Y ; E ; F)$. The principal symbol of $P, \sigma_{0}(P)$ is a section of $\operatorname{Hom}\left(\pi^{*} E, \pi^{*} F\right)$ homogeneous of degree 0 , i.e.

$$
\sigma_{0}(P)(Y, \lambda \xi)=\sigma_{0}(P)(x, \xi) \text { for } \lambda \in \mathbb{R}_{+} .
$$

The operator is elliptic if the homomorphism

$$
\sigma_{0}(P)(x, \xi): E_{x} \longrightarrow F_{x}
$$

is invertible for all nonzero $\xi$. In this case $P: L^{2}(Y ; E) \rightarrow L^{2}(Y ; F)$ is a Fredholm operator. As usual we define its analytic index to be

$$
\operatorname{Ind}(P)=\operatorname{dim} \operatorname{ker} P-\operatorname{dim} \operatorname{coker} P \text {. }
$$

The Atiyah-Singer index theorem identifies this index with a topological invariant constructed from the triple $\left[E, F, \sigma_{0}(P)\right]$.

Let $\widehat{T^{*} Y}$ be one point fiber compactification of $T^{*} Y$, that is we add one point to each fiber of $T^{*} Y$. The compactified space

$$
\widehat{\pi}: \widehat{T^{*} Y} \longrightarrow Y
$$

is a fiber bundle with fiber $S^{n}$. It is useful to have a second description of this space. Fix a Riemannian metric $g$ on $T^{*} Y$ and define the co-ball bundle

$$
B^{*} X=\left\{(x, \xi) \in T^{*} Y:\|\xi\| \leq 1\right\} .
$$


The boundary of the co-ball bundle is the unit co-sphere bundle, $S_{g}^{*} Y$. Two copies of the co-ball bundle glued along their boundaries produces a manifold which is diffeomorphic to $\widehat{T^{*} Y}$

$$
\widehat{T^{*} Y} \simeq B^{*} Y \bigsqcup_{S_{g}^{*} Y} B^{*} Y
$$

For notational convenience we label the co-ball bundles $B_{ \pm}^{*} Y$. One can think of $B_{+}^{*} Y$ as the interior of the unit ball in $\widehat{T^{*} Y}$ and $B_{-}^{*} Y$ as the exterior of the unit sphere. If $\omega$ is the symplectic form on $T^{*} Y$ then $\pm \omega^{n}$ define orientations for $B_{ \pm}^{*} Y$ which glue together to define an orientation on $\widehat{T^{*} Y}$.

Using the symbol of the operator we construct a vector bundle over $V_{P} \rightarrow \widehat{T^{*} Y}$ as follows:

$$
V_{P}\left\lceil_{B_{+}^{*} Y}=\pi^{*}(E), \quad V_{P}\left\lceil_{B_{-}^{*} Y}=\pi^{*}(F) .\right.\right.
$$

To complete the construction we use the symbol of $P$ to glue the two pieces together along their common boundary, the co-sphere bundle, by the equivalence relation:

$$
(x, \xi, e) \simeq(x, \xi, f) \text { if } f=\sigma_{0}(P)(x, \xi) e .
$$

Here we use the fact that $\sigma_{0}(P)(x, \xi)$ is an isomorphism for each $\xi \neq 0$.

Remark 4. The bundle $V_{P}$ is homotopy theoretic object, i.e. the interesting geometric invariants of $V_{P}$ depend only on the homotopy classes of $E, F$ and $\sigma_{0}(P)$. For example: If $P_{t}$ is a continuous, one parameter family of elliptic operators in $\Psi_{\mathrm{KN}}^{0}(Y ; E, F)$ then the bundles $V_{P_{t}}$ are all equivalent. The important data in $V_{P}$ is described by the class it defines in $K_{0}\left(\widehat{T^{*} Y}\right)$. The Atiyah-Index theorem identifies $\operatorname{Ind}(P)$ with a K-theoretic invariant of $V_{P}$.

The Chern character defines a ring homomorphism from

$$
\mathrm{Ch}: K_{0}\left(\widehat{T^{*} Y}\right) \longrightarrow H^{2 *}\left(\widehat{T^{*} Y}\right) \text {. }
$$

The index of $P$ can be expressed in terms of $\mathrm{Ch}\left(\left[V_{P}\right]\right)$ and the Todd class of $Y$ which we denote $\operatorname{Td}(Y) \in \oplus H_{\mathrm{dR}}^{2 i}(Y)$. For more details on this aspect of the subject see [19] or [25]. With these preliminaries we can state:

Theorem 8 (Atiyah-Singer index formula). If $P$ is an elliptic Kohn-Nirenberg pseudodifferential operator from $\mathcal{C}^{\infty}(Y ; E)$ to $\mathcal{C}^{\infty}(Y ; F)$ then $P$ is a Fredholm operator and

$$
\operatorname{Ind}(P)=\left\langle\widehat{\pi}^{*} \operatorname{Td}(Y) \wedge \operatorname{Ch}\left(V_{P}\right),\left[\widehat{T^{*} Y}\right]\right\rangle .
$$

This formula looks explicit, but in fact it is quite difficult to use it to compute the index of $P$ except in special cases. If the operator $P$ is of "Dirac type" then a much more explicit formula is available. We observe that $\operatorname{Ch}\left(V_{P}\right)$ is a quantity depending on $P$ whereas $\operatorname{Td}(Y)$ does not depend on the operator at all.

A Dirac operator is defined on a manifold with a spin structure. This is, in a certain sense a refinement of an orientation and there are topological obstructions to its existence. An equally explicit formula is available for a Dirac operator defined by a Spin- $\mathbb{C}$ structure. It turns out that an almost complex manifold, $Y$ always has a natural Spin- $\mathbb{C}$ structure. This in turn defines a Dirac operator which acts on the complex spinors. For the case of a complex manifold, the complex spinors can be identified with $S(Y)=\oplus_{q} \Lambda^{0, q} Y$ and the Spin- $\mathbb{C}$ Dirac operator with

$$
\varlimsup_{\mathbb{C}}=\bar{\partial}+\bar{\partial}^{*}: \mathcal{C}^{\infty}\left(Y ; \Lambda^{0, \text { even }} Y\right) \longrightarrow \mathcal{C}^{\infty}\left(Y ; \Lambda^{0, \text { odd }} Y\right) .
$$


The index of this operator is called the Todd genus of $Y$; it is expressed as a cohomological pairing on $Y$ as

$$
\operatorname{Ind}\left(\varlimsup_{\mathbb{C}}\right)=\left\langle\operatorname{Td}\left(T^{1,0} Y\right),[Y]\right\rangle .
$$

If $E \rightarrow Y$ is a complex vector bundle then, by choosing an Hermitian connection on $E$ one defines a twisted, Spin- $\mathbb{C}$ Dirac operator $\widetilde{\partial}_{E}$, acting on $\mathcal{C}^{\infty}(Y ; E \otimes S(Y))$. Again there is a formula for $\operatorname{Ind}\left(\varlimsup_{E}\right)$ as cohomological pairing on $Y$ :

$$
\operatorname{Ind}\left(\Im_{E}\right)=\left\langle\operatorname{Ch}(E) \wedge \operatorname{Td}\left(T^{1,0} Y\right),[Y]\right\rangle .
$$

Here, as above $\operatorname{Ch}(E)$ is the Chern character of the complex vector bundle $E \rightarrow Y$. While formula 38 looks quite similar to this formula, the latter formula is much simpler because: (1) The integration takes place on $Y$. (2) Only the Chern character of the bundle $E \rightarrow Y$ appears, the symbol of the Dirac operator does not appear explicitly. This special case of the Atiyah-Singer theorem suffices for most of our applications.

It is useful to have an explicit formula for the Chern character. Using the Chern-Weil theory it can be expressed in terms of the curvature of a connection defined on $E$, see [19] and [10]. Let $E$ be an hermitian, complex vector bundle and $\nabla_{E}$ a unitary connection on $E$. The curvature of the connection is $K_{E}=\nabla_{E}^{2}$; in terms of a local trivialization of $E$ it is represented by a matrix of 2-forms $\Omega_{E}$. Using the transformation formula for $\Omega_{E}$, under a change of local framing, as well as the invariance properties of the trace powers it is not difficult to show that

$$
\operatorname{Ch}(E)=\operatorname{Tr}\left(\exp \left[\frac{i}{2 \pi} \Omega_{E}\right]\right) .
$$

is globally defined. The Bianchi identity implies that it is closed. This sum of forms is a representative of the Chern character of $E$.

Remark 5. Let $X$ be a contact manifold with contact field $\mathcal{H}$. A choice of almost complex structure on the fibers of $\mathcal{H}$ defines the bundles $\Lambda_{b}^{0, q} X$. The Whitney sum

$$
S_{X}=\bigoplus_{q=0}^{n} \Lambda_{b}^{0, q} X
$$

defines a Spin- $\mathbb{C}$ structure on $X$. The $\bar{\partial}_{b}$-operator is defined by (14), though it does not define a complex unless the almost complex structure comes from an integrable CR-structure. In any case the operator

$$
\check{\partial}_{\mathbb{C}}=\bar{\partial}_{b}+\bar{\partial}_{b}^{*}: \mathcal{C}^{\infty}\left(X ; S_{X}\right) \longrightarrow \mathcal{C}^{\infty}\left(X ; S_{X}\right)
$$

is a self adjoint Spin- $\mathbb{C}$ Dirac operator.

\section{The Boutet De Monvel INDEX FORMUla}

Let $X$ denote a compact, contact manifold. We would like to prove a result, analogous to (38), for elliptic operators in $\Psi_{\mathrm{H}}^{*}(X ; E)$. A problem arises at the beginning of the discussion: The condition for a Heisenberg operator $A$ to be elliptic is that its principal symbol $\sigma_{m}^{\mathrm{H}}(A)$ be invertible in the isotropic algebra. This is not a pointwise condition in $T^{*} X$ but rather that there exist a symbol $\sigma_{-m}^{\mathrm{H}}(B)$ so that

$$
\sigma_{m}^{\mathrm{H}}(A)( \pm) \#_{ \pm} \sigma_{-m}^{\mathrm{H}}(B)( \pm)=\sigma_{-m}^{\mathrm{H}}(B)( \pm) \#_{ \pm} \sigma_{m}^{\mathrm{H}}(A)( \pm)=\mathrm{Id} .
$$

This condition is global in each fiber of the cotangent space.

Consider the following example. Let $S$ be a generalized Szegó projection (at level 0) and $\mu$ be a complex valued function on $X$. The operator

$$
A_{\mu}=\mathrm{Id}+\mu S
$$


is Fredholm if and only if $\mu$ does not assume that value -1 . Let

$$
B_{\mu}=\mathrm{Id}-\frac{\mu}{1+\mu} S \text {. }
$$

A simple calculation shows that

$$
B_{\mu} A_{\mu}=\mathrm{Id}+\frac{\mu}{1+\mu}[S, \mu] S .
$$

As noted above $[\mu, S] \in \Psi_{\mathrm{H}}^{-1}(X)$, thus $B_{\mu}$ is a left inverse up to a compact error. A similar calculation shows that $B_{\mu}$ is also a right inverse, up to a compact error. On the other hand, in appropriate coordinates the principal symbol of $A_{\mu}$ is given by

$$
\sigma_{0}^{\mathrm{H}}\left(A_{\mu}\right)(x, \sigma)= \begin{cases}1+\mu(x) \exp \left(-\frac{\left|\sigma^{\prime}\right|^{2}}{\sigma_{0}}\right) & \text { for } \sigma_{0}>0 \\ 1 & \text { for } \sigma_{0} \leq 0\end{cases}
$$

From this formula it is quite clear that if $\mu(x)$ is real and less than -1 then $\sigma_{0}^{\mathrm{H}}\left(A_{\mu}\right)(x, \sigma)$ vanishes on a hypersurface in $T_{x}^{*} X$. Nonetheless it follows from the composition formula for principal symbols that

$$
1=\sigma_{0}^{\mathrm{H}}(\mathrm{Id})( \pm)=\sigma_{0}^{\mathrm{H}}\left(A_{\mu}\right)( \pm) \#_{ \pm} \sigma_{0}^{\mathrm{H}}\left(B_{\mu}\right)( \pm)=\sigma_{0}^{\mathrm{H}}\left(B_{\mu}\right)( \pm) \#_{ \pm} \sigma_{0}^{\mathrm{H}}\left(A_{\mu}\right)( \pm) .
$$

Exercise 22. Provide the details of this computation.

Our approach is therefore somewhat indirect. In these notes we restrict our attention to Heisenberg operators of a fairly simple sort. We consider operators of the form $\mathrm{Id}+A^{\prime}$ where $A^{\prime} \in \Psi_{\mathrm{H}}^{0}(X ; E)$ and the "full" symbol of $A^{\prime}$ vanishes in the lower half of the cotangent bundle. In other words if we fix a Darboux coordinate system and express $A^{\prime}$ by the formula (23), then the function $\mathfrak{a}^{\prime}\left(x, \sigma_{0}, \sigma^{\prime}\right)$ vanishes identically for negative $\sigma_{0}$. This condition is coordinate invariant. Indeed operators with symbol vanishing in the lower half space are an ideal in the Heisenberg calculus. We denote this ideal by $\mathcal{I}_{+}^{0}(X ; E)$. The case of a general elliptic element is considerably more complicated; it is treated in [14]. To obtain a formula for the index of an operator of the form $\operatorname{Id}+A^{\prime}$ with $A^{\prime} \in \mathcal{I}_{+}^{0}(X ; E)$ we relate this index to the index of a generalized Toeplitz operator. Boutet de Monvel gave a formula for the index of a Toeplitz operator. Using Boutet's formula and a limiting argument one can obtain a cohomological formula for the index of $\mathrm{Id}+A^{\prime}$.

The next order of business therefore is Boutet de Monvel's formula for the index of a Toeplitz operator. Let $X$ be a contact manifold and $E \rightarrow X$ a complex vector bundle. We fix an adapted almost complex structure, $J$ on the contact field, $\mathcal{H}$ and let $S_{E}$ denote a generalized Szegó projection at level 0 with principal symbol $s_{0} \otimes \operatorname{Id}_{E}$. As before $s_{0}$ is the symbol of the projection onto the ground state of the field of harmonic oscillators defined by the choice of almost complex structure. Given a section $\alpha \in \mathcal{C}^{\infty}(X ; \operatorname{Hom}(E))$, let $T_{\alpha}=S_{E} \alpha S_{E}$ denote the Toeplitz operator it defines. If $\alpha$ is everywhere invertible then the operator $T_{\alpha}$ is Fredholm from the range $S_{E}$ to itself. The proof used in the scalar case works, mutatis mutandis. Traditionally one would say that $\alpha$ is the symbol of the Toeplitz operator. Using this symbol we construct a vector bundle over $X \times S^{1}$ which we denote by $E_{\alpha}$. The base space, $X \times S^{1}$ is identified with $X \times[0,1] / \sim$ where $(x, 0) \sim(x, 1)$. We glue the ends of $X \times[0,1] \times E$ together using the isomorphism $\alpha$ :

$$
(x, 0 ; e) \sim(x, 1 ; \alpha(x) e) .
$$

This defines the bundle $E_{\alpha} \rightarrow X \times S^{1}$.

The manifold $X=X \times S^{1}$ has a natural homotopy class of almost complex structures. Since $T X=\mathcal{H} \oplus \mathbb{R} T$, we have $T X=\mathcal{H} \oplus \mathbb{R} T \oplus T S^{1}$. Let $\partial_{t}$ denote a coordinate vector field tangent to $S^{1}$. We extend the almost complex structure defined on $\mathcal{H}$ by letting 
$J T=\partial_{t}$. A manifold with an almost complex structure has a natural Spin- $\mathbb{C}$ structure. This defines a Dirac operator $\partial_{E_{\alpha}}$ acting $\Lambda^{0 \text {,even }} X \otimes E_{\alpha}$ In [14] it is shown that the index of the Toeplitz operator $T_{\alpha}$ equals that of the twisted, Spin- $\mathbb{C}$ Dirac operator $\partial_{E_{\alpha}}$. From (39) we deduce:

Theorem 9 (Boutet de Monvel index formula for a Toeplitz operator). Let $E \rightarrow X$ be a complex vector bundle and $\alpha \in \mathcal{C}^{\infty}(X ; \operatorname{hom}(E))$ which is everywhere invertible. Let $S_{E}$ denote the Szegő projector acting on sections of $E$. The Toeplitz operator $T_{\alpha}=S_{E} \alpha S_{E}$ is Fredholm with

$$
\operatorname{Ind}\left(T_{\alpha}\right)=\operatorname{Ind}\left({\check{E_{\alpha}}}\right)=<\operatorname{Ch}\left(E_{\alpha}\right) \wedge \operatorname{Td}\left(T^{1,0} X\right),[X]>\text {. }
$$

In the special case of $X=\partial D, D \subset \mathbb{C}^{n}$, a strictly pseudoconvex domain and $E \rightarrow X$ a trivial bundle the formula simplifies considerably. In this case the Todd class is trivial. For $\mu \in \mathcal{C}^{\infty}(X$; hom $E)$, a bundle isomorphism, we have the index formula

$$
\operatorname{Ind}\left(S_{E} \mu S_{E}\right)=c_{n} \int_{X} \operatorname{Tr}\left[\mu^{-1} d \mu\right]^{2 n+1} .
$$

Here $c_{n}$ is a universal constant, depending only on the dimension. From this formula it follows that the index is zero if fiber dimension of $E$ is less than $(\operatorname{dim} X+1) / 2$.

\section{AN INDEX THEOREM FOR THE HEISENBERG CALCULUS}

Now we turn to the computation of the index of a Heisenberg-elliptic operator of the form

$$
A=\operatorname{Id}_{E}+A^{\prime}
$$

where $A^{\prime} \in \mathcal{I}_{+}^{0}(X ; E)$. To compute the $\operatorname{Ind}(A)$ we fix an adapted almost complex structure $J$ on the contact field. Let $h_{J}$ denote the field of harmonic oscillators fixed by this choice. Let $\left\{s_{k}: k \in \mathbb{N}_{0}\right\}$ denote the symbols of the projectors onto the eigenspaces of $h_{J}$. These symbols satisfy the relations

$$
s_{k} \# h_{J}=h_{J} \# s_{k}=(n+2 k) s_{k} \text { and } s_{k} \# s_{j}=s_{j} \# s_{k}=\delta_{j k} s_{k} .
$$

The \#-product refers to the \#-product defined on $\sigma_{0}=1$ by the symplectic form dual to $d \theta$. For each $N \geq 0$ we let $S_{E}^{(N)} \in \Psi_{\mathrm{H}}^{0}(X ; E)$ denote a choice of generalized Szegó projectors to level $N$ acting on sections of $E$. Recall that this means

$$
\left[S_{E}^{(N)}\right]^{2}=S_{E}^{(N)}, \quad \sigma_{0}^{\mathrm{H}}\left(S_{E}^{(N)}\right)(+)=\sum_{j=0}^{N} s_{j} \otimes \operatorname{Id}_{E}=s_{E}^{(N)} .
$$

These operators belong to $\mathcal{I}_{+}^{0}(X ; E)$. Without loss of generality we can assume that these projections are orthogonal with respect to a fixed volume form on $X$ and hermitian metric on $E$. The advantage of working with elements of $\Psi_{\mathrm{H}}^{*}(X ; E)$ of this special form is the following result:

Theorem 10. If $A^{\prime} \in \mathcal{I}_{+}^{0}(X ; E)$ and $\operatorname{Id}_{E}+A^{\prime}$ is a Fredholm operator then, for sufficiently large $N$ the generalized Toeplitz operator to level $N, S_{E}^{(N)}\left(\operatorname{Id}_{E}+A^{\prime}\right) S_{E}^{(N)}$ is Fredholm and

$$
\operatorname{Ind}\left(\operatorname{Id}_{E}+A^{\prime}\right)=\operatorname{Ind}\left(S_{E}^{(N)}\left(\operatorname{Id}_{E}+A^{\prime}\right) S_{E}^{(N)}\right)
$$

The index on the left is of the operator

$$
\operatorname{Id}_{E}+A^{\prime}: L^{2}(X ; E) \longrightarrow L^{2}(X ; E)
$$

whereas the index on the right is of the generalized Toeplitz operator to level $N$ acting on $S_{E}^{(N)} L^{2}(X ; E)$. 
Proof. For each $N$ we have the identity:

$$
\begin{gathered}
\operatorname{Id}_{E}+A^{\prime}=S_{E}^{(N)}\left(\operatorname{Id}_{E}+A^{\prime}\right) S_{E}^{(N)}+\left(\operatorname{Id}_{E}-S_{E}^{(N)}\right)+ \\
S_{E}^{(N)} A^{\prime}\left(\operatorname{Id}_{E}-S_{E}^{(N)}\right)+\left(\operatorname{Id}_{E}-S_{E}^{(N)}\right) A^{\prime} S_{E}^{(N)}+\left(\operatorname{Id}_{E}-S_{E}^{(N)}\right) A^{\prime}\left(\operatorname{Id}_{E}-S_{E}^{(N)}\right) .
\end{gathered}
$$

Since $A^{\prime} \in \mathcal{I}_{+}^{0}(X ; E)$ it is not difficult to show that

$$
\sigma_{0}^{\mathrm{H}}\left(A^{\prime}\right) \#\left(\operatorname{Id}_{E}-s_{E}^{(N)}\right) \text { and }\left(\operatorname{Id}_{E}-s_{E}^{(N)}\right) \# \sigma_{0}^{\mathrm{H}}\left(A^{\prime}\right)
$$

tend to zero as symbols of order 0 . In fact, for large enough $N$ and $t \in[0,1]$ the operators

$$
\begin{gathered}
A_{t}=S_{E}^{(N)}\left(\operatorname{Id}_{E}+A^{\prime}\right) S_{E}^{(N)}+\left(\operatorname{Id}_{E}-S_{E}^{(N)}\right)+ \\
t\left[S_{E}^{(N)} A^{\prime}\left(\operatorname{Id}_{E}-S_{E}^{(N)}\right)+\left(\operatorname{Id}_{E}-S_{E}^{(N)}\right) A^{\prime} S_{E}^{(N)}+\left(\operatorname{Id}_{E}-S_{E}^{(N)}\right) A^{\prime}\left(\operatorname{Id}_{E}-S_{E}^{(N)}\right)\right]
\end{gathered}
$$

are all Fredholm. As the family depends smoothly on $t$ in the operator norm it is evident that $\operatorname{Ind}\left(A_{0}\right)=\operatorname{Ind}\left(A_{1}\right)$. In other words:

$$
\begin{aligned}
\operatorname{Ind}\left(\operatorname{Id}_{E}+A^{\prime}\right) & =\operatorname{Ind}\left(S_{E}^{(N)}\left(\operatorname{Id}_{E}+A^{\prime}\right) S_{E}^{(N)}+\left(\operatorname{Id}_{E}-S_{E}^{(N)}\right)\right) \\
& =\operatorname{Ind}\left(S_{E}^{(N)}\left(\operatorname{Id}_{E}+A^{\prime}\right) S_{E}^{(N)}\right) .
\end{aligned}
$$

The last index is of a generalized Toeplitz operator acting on $S_{E}^{(N)} L^{2}(X ; E)$.

Exercise 23. If $\mu$ is a complex valued function and $S$ is a level 0 Szegó projector, then

$$
\operatorname{Ind}(\operatorname{Id}+\mu S)=\operatorname{Ind}(S(\operatorname{Id}+\mu) S)
$$

provided that $\mu \neq-1$ at any point. This can be proved by directly comparing kernels and co-kernels

Exercise 24. If $\mu \in \mathcal{C}^{\infty}(X ; \operatorname{hom}(E))$ and $\operatorname{Id}_{E}+\mu$ is invertible at every point of $X$ then $\operatorname{Id}_{E}+\mu S_{E}$ is Fredholm. In case we have:

$$
\operatorname{Ind}\left(\operatorname{Id}_{E}+\mu S_{E}\right)=\operatorname{Ind}\left(S_{E}\left(\operatorname{Id}_{E}+\mu\right) S_{E}\right)
$$

Theorem 10 reduces the problem of computing $\operatorname{Ind}\left(\operatorname{Id}_{E}+A^{\prime}\right)$ to that of computing the index of a Toeplitz operator to level $N$, for large $N$ and then showing that the resultant formula has a reasonable limit as $N \rightarrow \infty$. Theorem 9 only gives the formula for the index of a Toeplitz operator at level 0. In fact we can replace $S_{E}^{(N)}$ with a Szegó projector at level 0 acting on a larger vector bundle. To accomplish this and thereby compute the index of the approximating Toeplitz operators we need to understand the symbols $\left\{s_{k}: k>0\right\}$ in greater detail. Indeed it suffices to do this analysis in the model case of $\mathbb{R}^{2 n}$ with its standard symplectic and complex structures. The transition to the contact manifold case follows exactly as in the construction of the bundle of isotropic algebras.

\section{THE STRUCTURE OF THE HIGHER EIGEN-PROJECTIONS}

If $\omega$ is the standard symplectic structure on $\mathbb{R}^{2 n}$ then the \#-product on isotropic symbols is given by the oscillatory integral

$$
a \# b(w)=\frac{1}{\pi^{2 n}} \iint a(w+u) b(w+v) e^{2 i \omega(u, v)} d u d v .
$$

If $a$ and $b$ belong to Schwartz class then this is an absolutely convergent integral. If $a \in S_{\text {iso }}^{m}\left(\mathbb{R}^{2 n}\right)$ and $b \in S_{\text {iso }}^{m^{\prime}}\left(\mathbb{R}^{2 n}\right)$ then $a \# b \in S_{\text {iso }}^{m+m^{\prime}}\left(\mathbb{R}^{2 n}\right)$ and it has the following asymptotic expansion:

$$
\left.a \# b \sim \sum_{k \geq 0} \frac{2^{k} i^{k}}{k !}\left(D_{x} \cdot D_{\eta}-D_{y} \cdot D_{\xi}\right)^{k} a(x, \xi) b(y, \eta)\right|_{x=y, \xi=\eta} .
$$


It is well known that if either $a$ or $b$ is a polynomial then this sum is finite and gives an exact formula for $a \# b$, see [21].

The differential operator appearing in this formula can be re-expressed in terms of the standard $(1,0)$ - and $(0,1)$-vector fields on $\mathbb{R}^{2 n}$ :

$$
\begin{aligned}
D_{x} \cdot D_{\eta}-D_{y} \cdot D_{\xi} & =\frac{1}{2}\left[\left(D_{x}-i D_{\xi}\right) \cdot\left(D_{y}+i D_{\eta}\right)-\left(D_{x}+i D_{\xi}\right) \cdot\left(D_{y}-i D_{\eta}\right)\right] \\
& =\partial_{z} \cdot \partial_{\bar{w}}-\partial_{\bar{z}} \cdot \partial_{w} .
\end{aligned}
$$

From this formula it is apparent that if $a$ and $b$ are both holomorphic (or both anti-holomorphic) polynomials, then only the $k=0$ term in (41) is non-zero. This shows that the holomorphic and anti-holomorphic polynomials are subalgebras in the \#-product structure. In fact the \#-product and ordinary pointwise product agree on these subalgebras. Let $z_{j}=x_{j}-i \xi_{j}$ and $\bar{z}_{j}=x_{j}+i \xi_{j}$. Let $\mathcal{I}_{k}$ denote the set of multi-indices of length $k$ and $W_{k}$ and $\bar{W}_{k}$ denote linear spans of $\left\{z^{\alpha}: \alpha \in \mathcal{I}_{k}\right\}$ and $\left\{\bar{z}^{\alpha}: \alpha \mid \in \mathcal{I}_{k}\right\}$ respectively. Note that as vector spaces

$$
W_{k} \simeq W_{k} \# s_{0} \text { and } \bar{W}_{k} \simeq s_{0} \# \bar{W}_{k}
$$

Given the symplectic form $\omega$ we define a linear functional on $\mathcal{S}\left(\mathbb{R}^{2 n}\right)$ by setting

$$
\operatorname{Tr}(a) \int_{\mathbb{R}^{2 n}} a \omega^{n}
$$

Exercise 25. If $a \in \mathcal{S}\left(\mathbb{R}^{2 n}\right)$ show that $q_{W}(a)$ is a trace class operator and

$$
\operatorname{Tr}(a)=\operatorname{Tr}\left(q_{W}(a)\right) .
$$

Exercise 26. Show that if $a, b \in \mathcal{S}\left(\mathbb{R}^{2 n}\right)$ then prove directly that

$$
\operatorname{Tr}(a \# b)=\operatorname{Tr}(b \# a) .
$$

This shows that $\mathrm{Tr}$ behaves like a trace on this algebra.

Using the trace functional we define inner products on the vector spaces $W_{k}$ :

$$
\langle p, q\rangle=\operatorname{Tr}\left(s_{0} \# \bar{p} \# q \# s_{0}\right) .
$$

The symbol of the harmonic oscillator is

$$
h(x, \xi)=|x|^{2}+|\xi|^{2} .
$$

We have the identities:

$$
h=\sum_{j=1}^{n} z_{j} \# \bar{z}_{j}+n,\left[z_{i}, z_{j}\right]=-2 \delta_{i j},\left[h, z_{i}\right]=2 z_{i} .
$$

The projection on the ground state of the harmonic oscillator has symbol $s_{0}=2^{n} e^{-h}$. It satisfies the symbolic identities:

$$
h \# s_{0}=s_{0} \# h=n s_{0}, \quad s_{0} \# s_{0}=s_{0}, \overline{s_{0}}=s_{0} .
$$

The symbol of the projection onto the eigenspace with eigenvalue $n+2 k$ is $s_{k}$ and it satisfies

$$
h \# s_{k}=(n+2 k) s_{k}, \quad s_{k} \# s_{k}=s_{k}=\overline{s_{k}} .
$$

These identities are easily proved using the quantization of the symbols. Let

$$
C_{j}=q_{W}\left(\frac{i}{2} \bar{z}_{j}\right), \quad j=1, \ldots, n
$$


denote the annihilation operators defined in (31) and $v_{0}$ the ground state for $\mathbf{H}_{n}=q_{W}(h)$. As noted above the eigenspace of $\mathbf{H}_{n}$ with eigenvalue $n+2 k$ is spanned by:

$$
\left\{C^{* \alpha} v_{0}:|\alpha|=k\right\} \text {. }
$$

Moreover,

$$
\left\langle C^{* \alpha} v_{0}, C^{* \beta} v_{0}\right\rangle=c_{\alpha} \delta_{\alpha \beta} .
$$

Exercise 27. Prove these formulæ using integration by parts and the commutator relations.

Using these facts we obtain the following formula for $s_{k}$.

$$
s_{k}=\sum_{\alpha \in \mathcal{I}_{k}} \frac{z^{\alpha} \# s \# \bar{z}^{\alpha}}{c_{\alpha}} .
$$

As the eigenspaces of $\mathbf{H}_{n}$ are orthogonal we have $s_{k} \# s_{l}=0$ if $k \neq 0$. If we let

$$
s^{(N)}=\sum_{k \leq N} s_{k} \text { then } s^{(N)} \# s^{(N)}=s^{(N)} .
$$

Thus $s^{(N)}$ is the symbol of the projection to level $N$.

To complete our discussion we define the vector valued symbols

$$
q_{k}=\left(\frac{s_{0} \# \bar{z}^{\alpha}}{\sqrt{c_{\alpha}}}: \alpha \in \mathcal{I}_{k}\right) .
$$

If $f=p \# s_{0} \in W_{k} \# s_{0}$ then $q_{k} \# f$ is $s_{0}$ times the vector of coefficients of $p$ with respect to the orthonormal basis $\left\{z^{\alpha}\left[c_{\alpha}\right]^{-\frac{1}{2}}: \alpha \in \mathcal{I}_{k}\right\}$. That is $q_{k}$ defines an isomorphism from $W_{k} \# s_{0} \subset \mathcal{S}\left(\mathbb{R}^{2 n}\right)$ to $\mathbb{C}^{\left|I_{\alpha}\right|}$. These vector valued symbols satisfy the identities:

$$
\begin{aligned}
q_{l} \# q_{k}^{*} & =\delta_{l k} \operatorname{Id}_{W_{l}} \otimes s_{0}, \\
q_{k}^{*} \# q_{k} & =s_{k} .
\end{aligned}
$$

If $a \in \mathcal{S}\left(\mathbb{R}^{2 n}\right)$ then

$$
\begin{aligned}
s^{(N)} \# a \# s^{(N)} & =\sum_{k, l=0}^{N} \sum_{\alpha \in \mathcal{I}_{k}} \sum_{\beta \in \mathcal{I}_{l}} \frac{1}{c_{\alpha} c_{\beta}}\left(z^{\alpha} \# s_{0} \# \bar{z}^{\alpha} \# a \# z^{\beta} \# s_{0} \# \bar{z}^{\beta}\right) \\
& =\sum_{\alpha \in \mathcal{I}_{k}} \sum_{\beta \in \mathcal{I}_{l}} a_{\alpha \beta} \frac{1}{\sqrt{c_{\alpha} c_{\beta}}}\left(z^{\alpha} \# s_{0} \# \bar{z}^{\beta}\right)
\end{aligned}
$$

where

$$
a_{\alpha \beta}=\operatorname{Tr}\left[\frac{1}{\sqrt{c_{\alpha} c_{\beta}}}\left(s_{0} \# \bar{z}^{\alpha} \# a \# z^{\beta} \# s_{0}\right)\right]
$$

Let

$$
W^{(N)}=\bigoplus_{k=0}^{N} W_{k} \# s_{0} \text { and } q^{(N)}=\bigoplus_{k=0}^{n} q_{k},
$$

from the remarks above it is clear that we can interpret the matrix $a^{(N)}=a_{\alpha \beta}$ as an element of $\operatorname{hom}\left(W^{(N)}\right)$. Using the relations in (44) we see that

$$
q^{(N)} \# s^{(N)} \# a \# s^{(N)} \# q^{(N) *}=a^{(N)} \otimes s_{0} .
$$

Symbols $a \in \mathcal{S}\left(\mathbb{R}^{2 n} ; \operatorname{hom}(V)\right)$ for a complex vector space, $V$ are treated analogously. The matrices $\left\{a^{(N)}\right\}$ are then homomorphisms of $V \otimes W^{(N)}$.

These considerations are easily transferred to the context of a contact manifold $X$ with a choice of adapted almost complex structure. Indeed one can simply regard this discussion as taking place in a single fiber of the cotangent space. The data then varies smoothly as the base point varies. A few additional remarks should clarify this construction. The vector spaces $W_{k}$ are canonically defined by the choice of almost complex structure and therefore 
define global vector bundles over $X$. We denote these vector bundles by $\mathcal{W}_{k}$. Complex multiples of the globally defined symbol $s_{0}$ then define a trivial line bundle; the bundles $\mathcal{W}_{k} \otimes s_{0}$ are also globally defined and isomorphic to $\mathcal{W}_{k}$. The symbols $\left\{q_{k}\right\}$ are globally defined and take values in hom $\left(\mathbb{C}, \mathcal{W}_{k} \otimes s_{0}\right)$. When quantized they define operators from $\mathcal{C}^{\infty}(X)$ to $\mathcal{C}^{\infty}\left(X ; \mathcal{W}_{k} \otimes s_{0}\right)$. Formula (47) implies that $s^{(N)} \#\left(1+a^{\prime}\right) \# s^{(N)}$ can be interpreted as the symbol of a Toeplitz operator at level zero acting on sections of the vector bundle with fiber $\mathcal{W}^{(N)}=\oplus_{k=0}^{N} \mathcal{W}_{k}$. In order to treat an operator acting on sections of a vector bundle $E \rightarrow X$ we replace $\left\{\mathcal{W}_{k} \otimes s_{0}\right\}$ by $\left\{E \otimes \mathcal{W}_{k} \otimes s_{0}\right\}$ and $\left\{q_{k}\right\}$ by $\left\{\operatorname{Id}_{E} \otimes q_{k}\right\}$.

For a complex vector bundle $F$ let $S_{E \otimes F}$ denote a level 0 Szegő projector acting on $\mathcal{C}^{\infty}(X ; E \otimes F)$. Quantize the symbols $\left\{\operatorname{Id}_{E} \otimes q_{k}\right\}$ in the Heisenberg calculus to obtain operators

$$
Q_{k}^{\prime}: \mathcal{C}^{\infty}(X ; E) \rightarrow \mathcal{C}^{\infty}\left(X ; E \otimes \mathcal{W}_{k}\right)
$$

From the relations in (44) it follows that

$$
\begin{aligned}
\sigma_{0}^{\mathrm{H}}\left(Q_{k}^{\prime} Q_{l}^{\prime *}\right) & =\delta_{k l} \sigma_{0}^{\mathrm{H}}\left(S_{E \otimes \mathcal{W}_{k} \otimes s_{0}}\right), \\
\sigma_{0}^{\mathrm{H}}\left(Q_{k}^{\prime *} Q_{k}^{\prime}\right) & =\operatorname{Id}_{E} \otimes s_{k} .
\end{aligned}
$$

As these are principal symbol calculations they remain valid if we replace $Q_{k}^{\prime}$ by

$$
Q_{l}=S_{E \otimes \mathcal{W}_{l}} Q_{l}^{\prime} S_{l, E}
$$

From these symbolic calculations it follows easily that

$$
Q^{(N)}=\oplus_{l=0}^{N} Q_{l}
$$

is a Fredholm map from range $S_{E}^{(N)} \rightarrow$ range $S_{E \otimes \mathcal{W}^{(N)} \text {. }}$

The operator $Q^{(N)} S_{E}^{(N)}\left(\operatorname{Id}_{E}+A^{\prime}\right) S_{E}^{(N)} Q^{(N)^{*}}$ is a level 0 Toeplitz operator acting on $\mathcal{C}^{\infty}\left(X ; E \otimes \mathcal{W}^{(N)}\right)$. The computations above imply that

$$
\sigma_{0}^{\mathrm{H}}\left(Q^{(N)} S_{E}^{(N)}\left(\operatorname{Id}_{E}+A^{\prime}\right) S_{E}^{(N)} Q^{(N)^{*}}\right)=\sigma_{0}^{\mathrm{H}}\left(S_{E \otimes \mathcal{W}^{(N)}} a^{(N)} S_{E \otimes \mathcal{W}^{(N)}}\right)
$$

where $a^{(N)} \in \mathcal{C}^{\infty}\left(X ; \operatorname{Hom}\left(E \otimes \mathcal{W}^{(N)}\right)\right.$ is defined (fiberwise) in (45) and (46). From the formulæ (7) and (6), Theorem 10 and (50) it follows that, for large $N$, we have the identities

$$
\begin{aligned}
\operatorname{Ind}\left(\operatorname{Id}_{E}+A^{\prime}\right) & =\operatorname{Ind}\left(S_{E}^{(N)}\left(\operatorname{Id}_{E}+A^{\prime}\right) S_{E}^{(N)}\right) \\
& =\operatorname{Ind}\left(Q^{(N)} S_{E}^{(N)}\left(\operatorname{Id}_{E}+A^{\prime}\right) S_{E}^{(N)} Q^{(N)^{*}}\right) \\
& =\operatorname{Ind}\left(S_{E \otimes \mathcal{W}^{(N)}} a^{(N)} S_{E \otimes \mathcal{W}^{(N)}}\right) .
\end{aligned}
$$

The operator on the last line is a level 0 Toeplitz operator, its index can be computed using (40). To carry through this computation and obtain a cohomological formula for the index requires a diuscussion of the differential geometry of the bundle of isotropic algebras and especially of the subbundles $\left\{\mathcal{W}_{k} \# s_{0}\right\}$. As a careful discussion would require many additional pages we limit ourselves to a few general remarks. A complete discussion can be found in [14].

One can find a connection $\nabla$ on the bundle of isotropic algebras with many useful properties. The connection is a derivation with respect to the \#-product structure:

$$
\nabla(a \# b)=(\nabla a) \# b+a \#(\nabla b) .
$$

The symbol of the harmonic oscillator and the entire functional calculus it generates are flat sections with respect to this connection. In particular

$$
\nabla s_{k}=0 \text { for } k=0,1, \ldots
$$


Finally the connection carries sections of the subbundles $\left\{\mathcal{W}_{k} \# s_{0}\right\}$ to sections of these subbundles. Taken together these properties allow a very explicit computation of the contribution of the symbol $\sigma_{0}^{\mathrm{H}}\left(A^{\prime}\right)$ to the index formula. For each $N$ the Chern character appearing in (40) takes the form

$$
\operatorname{Ch}\left(E_{a^{(N)}}\right)=\operatorname{Tr}\left(\exp \left[\frac{i}{2 \pi} \Omega_{E_{a^{(N)}}}\right]\right) .
$$

The curvature forms on the bundles $E_{a^{(N)}}$ are defined by restricting the curvature form from an infinite dimensional bundle over $X \times S^{1}$ defined by $\sigma_{0}^{\mathrm{H}}\left(\mathrm{Id}+A^{\prime}\right)$. The trace operation defined in (43) transplants to define a fibrewise trace on sections of $\mathcal{S}\left(H^{+}\right)$. Because $a^{\prime}=\sigma_{0}\left(A^{\prime}\right)$ is rapidly decreasing on the fibers of $H^{+}$it is not difficult to show that the expression in (52) has a limit as $N$ tends to infinity. In the limit the matrix trace in (52) is replaced by this algebra trace. For example the principal term is of the form

$$
c_{n} \operatorname{Tr}\left[\left(\operatorname{Id}_{E}+a^{\prime}\right)^{-1} \# \nabla a^{\prime}\right]^{2 n+1} .
$$

Here $\left(\operatorname{Id}_{E}+a^{\prime}\right)^{-1}$ is the inverse with respect to the \#-product. This is a straightforward computation as $\nabla a^{\prime}$ is a (hom $E$ )-valued, Schwartz class 1 -form on $H^{+}$. If we let $\mathrm{Ch}\left(a^{\prime}\right)$ denote the limiting Chern character then

$$
\operatorname{Ind}\left(\operatorname{Id}+A^{\prime}\right)=\left\langle\operatorname{Ch}\left(a^{\prime}\right) \wedge \operatorname{Td}\left(T^{1,0} X\right),[X]\right\rangle \text {. }
$$

Two sorts of difficulties arise in the analysis of general elliptic elements in the Heisenberg calculus: The first problem one encounters is with symbols that do not vanish to high order along the set $\left\{\sigma_{0}=0\right\}$. In this case the algebra trace used above to define $\operatorname{Ch}\left(a^{\prime}\right)$ no longer converges absolutely. Instead one uses a $\zeta$-function regularization to extend the trace to these symbols. Unfortunately this leads to additional terms in the index formula which are essentially Wodzicki residue traces. The final difficulty is with symbols that do not reduce to the identity on $\left\{\sigma_{0}=0\right\}$. These are reduced to the previous case using a $\mathrm{K}$-theoretic argument. The index formula for these last two classes of operators is a less explicit than for the case treated above. The interested reader is referred to the monograph [14].

Philosophically the proof in the general case shows that, insofar as index computations are concerned, the Heisenberg algebra (stably)retracts onto the subalgebra of Toeplitz operators. Using this principle the indices of many operators can be computed quite explicitly. We close this section with such an example. Let $\theta$ denote a contact form and $T$ the associated Reeb vector field. Let $J$ denote a choice of adapted almost complex structure on $\mathcal{H}$ and $L$ a self adjoint differential operator with principal symbol $h_{J}$. If $\left\{X_{1}, \ldots, X_{n}, Y_{1}, \ldots, Y_{n}\right\}$ is a symplecto-normal basis with respect to $J$ and $d \theta$ then locally

$$
L=-\sum_{j=1}^{n}\left[X_{j}^{2}+Y_{j}^{2}\right]+\text { l. o.t. . }
$$

Let $S$ denote a Szegó projector compatible with $J$.

For a positive integer $N$ let $a$ be a smooth $U(N)$-valued function defined on $X$. Define a differential operator acting on $\mathbb{C}^{N}$-valued functions by

$$
\mathcal{L}_{a}=(L+i n T) \otimes \operatorname{Id}_{\mathbb{C}^{N}}+i a \otimes T .
$$

It is not difficult to show that $\mathcal{L}_{a}$ is a Fredholm operator. Using symbolic computations in the Heisenberg algebra one can show that

$$
\operatorname{Ind}\left(\mathcal{L}_{a}\right)=\operatorname{Ind}\left(S \otimes \operatorname{Id}_{\mathbb{C}^{N}} M_{a} S \otimes \operatorname{Id}_{\mathbb{C}^{N}}\right),
$$


where the operator on the right is a Toeplitz operator acting on $\operatorname{range}\left(S \otimes \operatorname{Id}_{\mathbb{C}^{N}}\right)$. As a corollary of this formula we obtain:

Corollary 1. If $(X, \mathcal{H})$ is a compact contact manifold then there exist second order, subelliptic differential operators with non-zero index.

Remark 6. This result should be contrasted with the classical result that an elliptic differential operator on an odd dimensional manifold always has index zero.

\section{GRAUERT tUbes AND THE ATIYAH-Singer INDEX THEOREM}

Boutet de Monvel's proof of the index theorem for Toeplitz operators uses essentially the full Atiyah-Singer index theorem. However in [7] he outlines an method for reversing the direction of the argument, explaining how one could prove the Atiyah-Singer theorem from the index theorem for Toeplitz operators. In [14] we give a new proof of the Toeplitz index formula which reduces the computation of the Toeplitz index to the computation of the index of a twisted, Spin- $\mathbb{C}$ Dirac operator. For this special case, the Atiyah-Singer theorem can be proved by relatively elementary means, see [5]. In this section we give an overview of this proof of the Atiyah-Singer theorem.

Recall that in section 2 we defined the notion of a Grauert tube. Let $Y$ denote a real analytic manifold and $\pi: T^{*} Y \rightarrow Y$ the cotangent bundle. For the purposes of this discussion we think of the Grauert tubes as a nested family of neighborhoods,

$$
\left\{\mathcal{X}_{\epsilon}: 0<\epsilon<\epsilon_{0}\right\}
$$

of the zero section in $T^{*} Y$ with an adapted complex structure as described in section 2. In the adapted complex structure the zero section of $T^{*} Y$ and the fibers of $\pi$ are totally real submanifolds. The Grauert tubes are Stein manifolds.

Let $\Omega^{n}\left(\mathcal{X}_{\epsilon}\right)$ denote the holomorphic $(n, 0)$-forms on the Grauert tube. If $\omega \in \Omega^{n}\left(\mathcal{X}_{\epsilon}\right) \cap$ $\mathcal{C}^{\infty}\left(\overline{\mathcal{X}_{\epsilon}} ; \Lambda^{n, 0}\right)$ then its restriction to a fiber of $\pi$ is a complex $n$-form which can be integrated over the fiber, thus defining a map:

$$
P_{\epsilon}(\omega)(y)=\int_{\pi^{-1}(y) \cap \mathcal{X}_{\epsilon}} \omega .
$$

This map is a Fourier integral operator with complex phase. In [12] it is shown that there exists an $0<\epsilon_{1}<\epsilon_{0}$ such that

$$
P_{\epsilon}: \Omega^{n}\left(\mathcal{X}_{\epsilon}\right) \cap \mathcal{C}^{\infty}\left(\overline{\mathcal{X}_{\epsilon}} ; \Lambda^{n, 0}\right) \longrightarrow \mathcal{C}^{\infty}(Y)
$$

is an isomorphism if $\epsilon<\epsilon_{1}$. By considering holomorphic $(n, 0)$-forms with polynomial growth at $b \mathcal{X}_{\epsilon}$ one can extend $P_{\epsilon}$ as isomorphism onto $\mathcal{C}^{-\infty}(Y)$.

Let $\Omega^{n}\left(b \mathcal{X}_{\epsilon}\right)$ denote the boundary values of holomorphic $(n, 0)$-forms. Forms smooth up to the boundary have smooth boundary values whereas forms with polynomial growth have distributional boundary values. For $\eta \in \Omega^{n}\left(b \mathcal{X}_{\epsilon}\right)$ let $\omega_{\eta}$ denote its extension to $\mathcal{X}_{\epsilon}$ as a holomorphic $(n, 0)$-form. Using, for example, the Henkin-Ramirez kernel one can obtain an operator:

$$
G_{\epsilon}: \Omega\left(b \mathcal{X}_{\epsilon}\right) \longrightarrow \mathcal{C}^{-\infty}(Y)
$$

which satisfies

$$
G_{\epsilon}(\eta)=P_{\epsilon}\left(\omega_{\eta}\right) .
$$

From the result above for $P_{\epsilon}$ it follows that, for $\epsilon<\epsilon_{1}$, this map is an isomorphism and its restriction to smooth forms is an isomorphism onto $\mathcal{C}^{\infty}(Y)$. A simpler proof of this result has recently be found by Raul Tataru, see [31]. These operators are again FIOs with 
complex phase and satisfy very precise mapping properties with respect to $L^{2}$-Sobolev space.

If $E \rightarrow Y$ is a complex vector bundle then $E_{\epsilon}=\pi^{*}(E)\left\lceil_{\mathcal{X}}\right.$ is also a smooth complex vector bundle. Since $\mathcal{X}_{\epsilon}$ is a Stein manifold it follows from the Oka-Grauert Principle, see [28] that there is a unique holomorphic bundle $\mathcal{E}_{\epsilon} \rightarrow \mathcal{X}_{\epsilon}$ equivalent to $E_{\epsilon}$. Since we can obtain the holomorphic structure by restricting $\mathcal{E}_{\epsilon^{\prime}}$ to $\mathcal{X}_{\epsilon}$ for an $\epsilon<\epsilon^{\prime}<\epsilon_{0}$ it is clear that the holomorphic structure is smooth up to $b \mathcal{X}_{\epsilon}$. Let $\Omega^{n}\left(b \mathcal{X}_{\epsilon} ; \mathcal{E}_{\epsilon}\right)$ denote the boundary values of holomorphic $(n, 0)$-forms with values in $\mathcal{E}_{\epsilon}$. The method used to prove that $G_{\epsilon}$ is an isomorphism for $\epsilon<\epsilon_{1}$ applies mutatis mutandis to show that the analogous map

$$
G_{\epsilon, E}: \Omega^{n}\left(b \mathcal{X}_{\epsilon} ; \mathcal{E}_{\epsilon}\right) \longrightarrow \mathcal{C}^{-\infty}(Y ; E)
$$

is also an isomorphism for $\epsilon<\epsilon_{1}$. As before the restriction of $G_{\epsilon, E}$ to smooth $(n, 0)$-forms gives an isomorphism onto $\mathcal{C}^{\infty}(Y ; E)$. For such a bundle we let $\mathcal{S}_{\epsilon, E}$ denote the classical Szegó projector onto boundary values of holomorphic sections.

Let $F \rightarrow Y$ be a second complex vector bundle and $\mathcal{F}_{\epsilon}$ the holomorphic representatives of the restrictions of $\pi^{*}(F)$ and $\mathcal{S}_{\epsilon, F}$ the classical Szegó projector onto boundary values of holomorphic sections of $\mathcal{F}_{\epsilon}$. Finally let $A$ be a classical elliptic pseudodifferential operator:

$$
A: \mathcal{C}^{\infty}(Y ; E) \rightarrow \mathcal{C}^{\infty}(Y ; F)
$$

For purposes of index computations there is no loss in generality in assuming that $A$ is of order zero, let $a_{0} \in \mathcal{C}^{\infty}\left(T^{*} Y \backslash\{0\} ; \operatorname{hom}\left(\pi^{*}(E), \pi^{*}(Y)\right)\right)$ denote its principal symbol. We define a Toeplitz operator:

$$
T_{\epsilon, a_{0}}=\mathcal{S}_{\epsilon, F} M_{a_{0}} \mathcal{S}_{\epsilon, E}: \Omega^{n}\left(b \mathcal{X}_{\epsilon} ; \mathcal{E}_{\epsilon}\right) \longrightarrow \Omega^{n}\left(b \mathcal{X}_{\epsilon} ; \mathcal{F}_{\epsilon}\right)
$$

This is slightly different from what we considered before as we now allow the operator to act between sections of different vector bundles. On the other hand, the Szegó projectors are now defined by integrable, almost complex structures.

Using results in [16] one can show that for $\epsilon<\epsilon_{1}$

$$
A_{\epsilon}^{\prime}=G_{\epsilon, F} T_{\epsilon, a_{0}} G_{\epsilon, E}^{*}
$$

is a pseudodifferential operator with the same principal symbol as $A$. As $G_{\epsilon, E}$ and $G_{\epsilon, F}$ are isomorphisms we obtain:

Theorem 11. If $E \rightarrow Y$ and $F \rightarrow Y$ are complex vector bundles and $A \in \Psi_{\mathrm{KN}}^{0}(Y ; E, F)$ is an elliptic operator with principal symbol $a_{0}$ then for sufficiently small $\epsilon$ we have

$$
\operatorname{Ind}(A)=\operatorname{Ind}\left(T_{\epsilon, a_{0}}\right) .
$$

As before $\operatorname{Ind}\left(T_{\epsilon, a_{0}}\right)$ can also be identified with a relative index

$$
\operatorname{Ind}\left(T_{\epsilon, a_{0}}\right)=\mathrm{R}-\operatorname{Ind}\left(\mathcal{S}_{\epsilon, E}, a_{0}^{-1} \mathcal{S}_{\epsilon, F} a_{0}\right) .
$$

The theorem therefore has the satisfying philosophical consequence of identifying the indices of all elliptic operators with relative indices of generalized Szegó projectors. A cohomological formula for $\operatorname{Ind}\left(T_{\epsilon, a_{0}}\right)$ leads immediately to a formula for $\operatorname{Ind}(A)$. For the case $E=F$ such a formula is given in (40). The general case has been considered by Leichtnam, Nest and Tsygan, see [27]. 


\section{THE CONTACT DEGREE AND INDEX OF FIOS}

In this section $(X, \mathcal{H})$ denotes a compact, contact manifold. Recall that a diffeomorphism $\psi$, of $X$ is a contact map if

$$
\psi_{*} \mathcal{H}_{x}=\mathcal{H}_{\psi(x)} .
$$

We call an orientation preserving contact diffeomorphism which preserves the co-orientation of $\mathcal{H}$ a contact transformation. Let $\mathcal{M}(X, \mathcal{H})$ denote isotopy classes of contact transformations; this is the contact mapping class group. As a final application of the ideas presented above we define a homomorphism c-deg : $\mathcal{M}(X, \mathcal{H}) \rightarrow \mathbb{Z}$ and give a formula for c-deg which is analogous to (40). If $X=S^{*} Y$ for a compact manifold $Y$ then, using a construction similar to that used in the previous section, we can relate this integer to the index of a certain class of Fredholm Fourier integral operators. Most of these results can be found in [13].

Choose an adapted almost complex structure $J$, for the fibers of $\mathcal{H}$. Let $S$ denote a generalized Szegó projector with principal symbol defined by the field of vacuum states for the corresponding field of harmonic oscillators. If $\psi$ is a contact transformation then

$$
S_{\psi}=\left(\psi^{-1}\right)^{*} S \psi^{*}
$$

is also a generalized Szegó projector, though its principal symbol is in general different from that of $S$. We define the contact degree as follows

$$
\mathrm{c}-\operatorname{deg}(\psi)=\mathrm{R}-\operatorname{Ind}\left(S, S_{\psi}\right) \text {. }
$$

Using the stability properties of the Fredholm index described in Lemma 1, it follows immediately that $\mathrm{c}-\operatorname{deg}(\psi)$ depends only on the equivalence class of $\psi$ in $\mathcal{M}(X, \mathcal{H})$. Using the co-cycle relation (36) one can prove that c-deg $(\psi)$ does not depend on the choice of $S$ and that if $\psi$ and $\phi$ are two contact transformations then

$$
\mathrm{c}-\operatorname{deg}(\psi \circ \phi)=\mathrm{c}-\operatorname{deg}(\psi)+\mathrm{c}-\operatorname{deg}(\phi) .
$$

In other words c-deg $: \mathcal{M}(X, \mathcal{H}) \rightarrow \mathbb{Z}$ is a homomorphism.

Exercise 28. Prove this statement.

The next order of business is to find a formula for c-deg $(\psi)$. For an arbitrary pair $S, S^{\prime}$ of generalized Szegó projectors, the relative index is a very delicate invariant and the value of R-Ind $\left(S, S^{\prime}\right)$ is difficult to compute. However in the special case that $S^{\prime}=S_{\psi}$ the relative index is much more robust and is essentially a topological invariant. We use a construction similar to that used to relate the index of a Toeplitz operator to the index of a Dirac operator on a mapping torus.

Define the mapping torus

$$
Z_{\psi}=X \times[0,1] /(x, 0) \sim(\psi(x), 1) .
$$

Since $\psi$ is a contact transformation the bundle $\mathcal{H}$ lifts to define a co-dimension 2 subbundle $\mathcal{H}_{\psi} \subset T Z_{\psi}$. This bundle has a conformal symplectic structure and therefore we can define an almost complex structure $\mathcal{J}$ on $\mathcal{H}_{\psi}$. If $t$ denotes the parameter and $T$ a vector field tangent to the fibers of $Z_{\psi} \rightarrow S^{1}$ transverse to $\mathcal{H}_{\psi} \oplus \mathbb{R} \partial_{t}$ then we can extend the almost complex structure defined $\mathcal{H}_{\psi}$ to an almost complex structure defined on $T Z_{\psi}$ by letting

$$
\mathcal{J} T=\partial_{t} .
$$

The almost complex structure on $T Z_{\psi}$ defines a canonical Spin- $\mathbb{C}$ structure on $Z_{\psi}$. Let $ð_{\psi}$ denote the Spin- $\mathbb{C}$ Dirac operator defined by a choice of hermitian structure. We think of $\widetilde{\partial}_{\psi}$ as acting from even to odd spinors. 
In [13] the following result is proved

Theorem 12. If $\psi$ is a contact transformation of $(X, \mathcal{H})$ then

$$
\mathrm{c}-\operatorname{deg} \psi=\operatorname{Ind}\left(\check{\partial}_{\psi}\right) \text {. }
$$

To prove this result we introduce a 'resolution' of the range of $S$ which is an acyclic differential complex very similar to the $\bar{\partial}_{b}$-complex. Recall however that the almost complex structure $J$, on $\mathcal{H}$ is not required to be integrable. This complex defines a self adjoint, Fredholm Heisenberg pseudodifferential operator $D_{0}$. The same construction applied to $S_{\psi}$ leads to the operator $D_{1}=\left(\psi^{-1}\right)^{*} D_{0} \psi^{*}$ which is therefore isospectral to $D_{0}$. These operators can be put into a continuous family $D_{t}$. Since the ends are isospectral, the spectral flow of the family is well defined; denote it by $\operatorname{sf}\left(\left[D_{t}\right]\right)$. The first step is to show that

$$
\mathrm{R}-\operatorname{Ind}\left(S, S_{\psi}\right)=\operatorname{sf}\left(\left[D_{t}\right]\right) \text {. }
$$

The next problem is to deform the family $\left[D_{t}\right]$ to a family of Spin- $\mathbb{C}$ Dirac operators $\left[\widetilde{\partial}_{t}\right]$ through Fredholm operators. Technically this is the most challenging step. This is because the operators in the family $\left[D_{t}\right]$ while Fredholm are not even Heisenberg elliptic, whereas Dirac operators are classical elliptic differential operators. To control this very singular perturbation problem we introduction an extension of the Heisenberg calculus which includes both the classical calculus and the Heisenberg calculus as subalgebras. Once this is accomplished, it follows from the stability of the Fredholm index that

$$
\operatorname{sf}\left(\left[D_{t}\right]\right)=\operatorname{sf}\left(\left[ð_{t}\right]\right) .
$$

It is then a fairly standard result that

$$
\operatorname{sf}\left(\left[ð_{t}\right]\right)=\operatorname{Ind}\left(ð_{\psi}\right) .
$$

For the case that the $\operatorname{dim} X=3$ one can use the Atiyah-Singer index theorem and Hirzebruch signature formula to obtain a simple explicit formula for c-deg $(\psi)$. In this case

$$
T Z_{\psi}=\mathcal{H}_{\psi} \oplus \mathbb{1}^{2}
$$

Here 1 denotes a trivial line bundle. Using formula (18) in [13] we deduce that

$$
\begin{aligned}
\operatorname{Ind}\left(\check{\partial}_{\psi}\right) & =\frac{1}{4} p_{1}\left(T Z_{\psi}\right)\left[Z_{\psi}\right] \\
& =\frac{1}{12} \operatorname{sig}\left(Z_{\psi}\right),
\end{aligned}
$$

where $p_{1}$ is the first Pontryagin class and $\operatorname{sig}\left(Z_{\psi}\right)$ is the signature of the 4-manifold $Z_{\psi}$. Let $P: Z_{\psi} \rightarrow S^{1}$ be the canonical projection and set

$$
Z_{0}=P^{-1}([0, \pi]) \text { and } Z_{1}=P^{-1}([\pi, 2 \pi]) .
$$

The manifolds with boundary $Z_{0}$ and $Z_{1}$ are diffeomorphic and, as oriented manifolds,

$$
Z_{\psi} \simeq Z_{0} \bigsqcup_{\text {boundary }}-Z_{1}
$$

that is we reverse the orientation of $Z_{1}$. Let $\operatorname{sig}\left(Z_{0}\right)=\operatorname{sig}\left(Z_{1}\right)$ denote the index of the nondegenerate pairing defined by the cup product on the image of $H^{2}\left(Z_{i}, b Z_{i}\right)$ in $H^{2}\left(Z_{i}\right)$. The Novikov addition formula states that

$$
\operatorname{sig}\left(Z_{\psi}\right)=\operatorname{sig}\left(Z_{0}\right)-\operatorname{sig}\left(Z_{1}\right)=0,
$$

see $[2,3]$. This proves the following result

Theorem 13. If $X$ is a 3 -dimensional contact manifold then the contact degree is the zero homomorphism. 
Remark 7. I would like to thank Rafe Mazzeo for pointing out the connection, for this case between the contact degree and the signature and Dennis Sullivan telling me about the Novikov addition formula.

Remark 8. In [11] the relative index is defined for a pair of embeddable CR-structures defined on a 3-dimensional contact manifold. There it is shown that the index descends to define an invariant on the "Teichmuller" space of CR-structures. This is the space of equivalence classes of complex structures on the contact field where two structures are equivalent if one is the push forward of the other by a contact map isotopic to the identity. The contact mapping class group acts on this Teichmuller space. Theorem 13 implies that the relative index actually descends to define an invariant on the moduli space itself.

As a second application of Theorem 12 we show that the contact degree is related to the index of a certain class of Fourier integral operators. For this application we need to restrict $X=S^{*} Y$, for $Y$ a compact manifold. If $\psi: S^{*} Y \rightarrow S^{*} Y$ is a contact transformation then it defines a conic Lagrangian submanifold of $T^{*} Y \backslash\{0\} \times T^{*} Y \backslash\{0\}$. To see this identify $S^{*} Y$ with a unit cosphere bundle and extend the map $\psi: S_{g}^{*} Y \rightarrow S_{g}^{*} Y$ to be homogeneous of degree 1 . Denote this extension by $\Psi$. It is a canonical transformation of the cotangent bundle, its graph, $\Lambda_{\psi}$ is therefore a conic, Lagrangian submanifold. Such a submanifold defines a class of Fourier integral operators, see [20] or [9]. Roughly speaking an operator belongs to this class if the wave front set of its Schwartz kernel is contained in $\Lambda_{\psi}$. For example, pseudodifferential operators are among the Fourier integral operators defined by the identity map.

Let $\left\{\mathcal{X}_{\epsilon}\right\}$ denote the Grauert tubes introduced in the previous section. As noted in section $2, S^{*} Y$ is contact equivalent to $b \mathcal{X}_{\epsilon}$ for any $\epsilon<\epsilon_{0}$. Therefore a contact transformation of $S^{*} Y$ can be thought of as defining a contact transformation of $b \mathcal{X}_{\epsilon}$. Let $\psi: b \mathcal{X}_{\epsilon} \rightarrow b \mathcal{X}_{\epsilon}$ be such a map. We define an operator on $\mathcal{C}^{\infty}(Y)$ by setting

$$
F_{\psi} u=G_{\epsilon} \psi^{*} G_{\epsilon}^{*}(u) .
$$

This is a Fredholm Fourier integral operator associated to the Lagrangian submanifold $\Lambda_{\psi}$. In [13] the index of this operator is computed.

Theorem 14. If $\psi: S^{*} Y \rightarrow S^{*} Y$ is a contact transformation then

$$
\operatorname{Ind}\left(F_{\psi}\right)=\mathrm{c}-\operatorname{deg}(\psi) .
$$

As a corollary of this result and Theorem 13 we have

Corollary 2. If $\operatorname{dim} Y=2$ then $\operatorname{Ind}\left(F_{\psi}\right)=0$ for every contact transformation.

The detailed proofs of these results are in [13] and [14].

Remark 9. Thus far no example of a contact manifold $X$ and contact transformation $\psi$ : $X \rightarrow X$ such that c-deg $(\psi) \neq 0$ has been found.

\section{REFERENCES}

[1] V.I. Arnold, Mathematical methods in classical mechanics, GTM, vol. 60, Springer Verlag, Berlin and New York, 1978.

[2] M.F. Atiyah, V.K. Patodi, and I.M. Singer, Spectral asymmetry and Riemannian geometry, I, Math. Proc. Camb. Phil. Soc 77 (1975), 43-69.

[3] M.F. Atiyah and I.M. Singer, The index of elliptic operators, III, Ann. of Math. 87 (1968), 546-604.

[4] R. Beals and P. Greiner, Calculus on Heisenberg Manifolds, Annals of Mathematics Studies, vol. 119, Princeton University Press, 1988. 
[5] N. Berline, E. Getzler, and M. Vergne, Heat kernels and Dirac operators, Grundlehren der mathematischen Wissenschaften, vol. 298, Springer-Verlag, Berlin Heidelberg New York, 1992.

[6] L. Boutet de Monvel, Integration des equations Cauchy-Riemann induites formelles, Seminaire GoulaouicLions-Schwartz (1974/75), IX.1-IX.13.

[7] L. Boutet de Monvel, On the index of Toeplitz operators of several complex variables, Invent. Math. 50 (1979), 249-272.

[8] L. Boutet de Monvel and V. Guillemin, The spectral theory of Toeplitz operators, Ann. of Math. Studies, vol. 99, Princeton University Press, 1981.

[9] J. Brüning and V.W. Guillemin (Editors), Fourier integral operators, Springer-Verlag, Berlin, Heidelberg, New York, Tokyo, 1994.

[10] S.S. Chern, Complex Manifolds without Potential Theory, Van Nostrand Reinhold Co., New York, 1967.

[11] Charles L. Epstein, A relative index on the space of embeddable CR-structures, I, II, Annals of Math. 147 (1998), 1-59, 61-91, Erratum to part I, Ann. of Math. 154, (2001), 223-226.

[12] Charles L. Epstein and Richard Melrose, Shrinking tubes and the $\bar{\partial}$-Neumann problem, preprint, 1990.

[13] _ Contact degree and the index of Fourier integral operators, Math. Res. Letters 5 (1998), 363-381.

[14] Charles L. Epstein, Richard Melrose, and Gerardo Mendoza, The Heisenberg algebra, index theory and homology, preprint, 1999.

[15] Israel Gohberg, Seymour Goldberg, and Marinus A. Kaashoek, Classes of linear operators, vol. I and II, Birkhäuser, Basel-Bostob-Berlin, 1990.

[16] V.W. Guillemin, Toeplitz operators in n dimensions, Int. Eq. Op. Theory 7 (1984), 145-205.

[17] V.W. Guillemin and M. Stenzel, Grauert tubes and the homogeneous Monge-Ampere equation, J. Differential Geom., 1991.

[18] F. Reese Harvey and H. Blaine Lawson, On the boundaries of complex analytic varieties, Ann. of Math. (2) 106 (1977), 223-290.

[19] Friedrich Hirzebruch, Topological methods in algebraic geometry, Grundlehren der mathematishen Wissenschaften, vol. 131, Springer Verlag, 1978.

[20] L. Hörmander, Fourier integral operators, I, Acta Math. 127 (1971), 79-183.

[21] _ The analysis of linear partial differential operators, vol. 3, Springer-Verlag, Berlin, Heidelberg, New York, Tokyo, 1985.

[22] J.J. Kohn, The range of the tangential Cauchy-Riemann operator, Duke Math. J., 53 (1986), 525-545.

[23] J.J. Kohn and L. Nirenberg, Non-coercive boundary value problems, Comm. Pure Appl. Math. 18 (1965), 443-492.

[24] __ On the algebra of pseudo-differential operators, Comm. Pure Appl. Math. 18 (1965), 269-305.

[25] H. Blaine Lawson Jr. and Marie-Louise Michelson, Spin geometry, Princeton Mathematical Series, vol. 38, Princeton University Press, 1989.

[26] P.D. Lax, On factorization of matrix valued functions, Comm. Pure Appl. Math. 29 (1976), 683-688.

[27] Eric Leichtnam, Ryszard Nest and Boris Tsygan, Local formula for index of a Fourier Integral Operator, 2002, to appear Jour. of Diff. Geo.

[28] J. Leiterer, Holomorphic vector bundles and the Oka-Grauert principle, Several Complex Variables, IV (S.G. Gindikin and G.M. Khenkin, eds.), Encyclopedia of Mathematical Sciences, vol. 10, Springer Verlag, 1990.

[29] Laszlo Lempert and Robert Szoke, Global solutions of the homogeneous complex Monge-Ampre equation and complex structures on the tangent bundle of riemannian manifolds, Math. Ann. 290 (1991), 689-712.

[30] Fritz Noether, Über eine Klasse singulärer Integralgleichungen, Math. Ann. 82 (1921), 42-63.

[31] Raul Tataru, Ph.D. Thesis MIT, to appear.

[32] M.E. Taylor, Noncommutative microlocal analysis, part I, Mem. Amer. Math. Soc., vol. 313, AMS, 1984.

[33] _ Noncommutative harmonic analysis, Mathematical Surveys and Monographs, vol. 22, AMS, Providence, R.I., 1986.

$$
\text { (C) Charles L. Epstein, 1999,2002 All rights reserved }
$$

Department of Mathematics, University of Pennsylvania

E-mail address: cle@math. upenn.edu 